\title{
CROSS CURVATURE FLOW ON LOCALLY HOMOGENEOUS THREE-MANIFOLDS (II)*
}

\author{
XIAODONG $\mathrm{CAO}^{\dagger}$ AND LAURENT SALOFF-COSTE
}

\begin{abstract}
In this paper, we study the positive cross curvature flow on locally homogeneous 3-manifolds. We describe the long time behavior of these flows. We combine this with earlier results concerning the asymptotic behavior of the negative cross curvature flow to describe the two sided behavior of maximal solutions of the cross curvature flow on locally homogeneous 3-manifolds. We show that, typically, the positive cross curvature flow on locally homogeneous 3-manifold produce an Heisenberg type sub-Riemannian geometry.
\end{abstract}

Key words. Cross Curvature Flow (XCF), locally homogeneous 3-manifold.

AMS subject classifications. Primary 53C44; Secondary 58J35, 35B55

\section{Introduction.}

1.1. Evolution equations on homogeneous manifolds. Hamilton's Ricci flow ([Ham82]) is the best known example of a geometric evolution equation. One of the aims of such flows is to obtain metrics with special properties (in the case of the Ricci flow, Einstein metrics). Special cases arise when the metric is invariant under a group of transformations and this property is preserved by the flow. In particular, if the group of isometries of the original Riemannian structure is transitive, then the geometric evolution equation reduces to an ODE in the tangent space of an arbitrary fixed origin. The Ricci flow on locally homogeneous 3-manifolds was analyzed in [IJ92] and on some homogeneous 4-manifolds in [IJL06].

The cross curvature flow, or (XCF), was introduced by Chow and Hamilton [CH04] and depends on the choice of a sign (see Section 1.3 below) leading to two flows: $(+\mathrm{XCF})$ and $(-\mathrm{XCF})$. Chow and Hamilton conjectured that for any compact 3 -manifold that admits a metric with negative sectional curvature, the normalized positive cross curvature flow, started at such a metric, exists for all time and converges to a hyperbolic metric. In [CNSC08], we study the asymptotic behavior of the negative cross curvature flow (-XCF) on homogeneous 3-manifolds. In this paper, we complement the results of [CNSC08] by studying the asymptotic behavior of the positive cross curvature flow, or $(+\mathrm{XCF})$, on homogeneous 3-manifolds. In the homogeneous case, the local existence is not an issue and the negative and positive cross curvature flows can be seen as the same basic flow (say, (-XCF)) run either in the forward or backward direction. Putting together the results of [CNSC08] and of this paper, we will thus describe both the forward and backward asymptotic behaviors of the maximal solution of (-XCF) through any given metric $g_{0}$ on any locally homogeneous 3-manifold.

Although we will give much more precise statements, the spirit of the main results proved in this paper is captured in the following theorem.

TheOrem 1.1. Let $\left(M, g_{0}\right)$ be a complete locally homogeneous 3-manifold (compact or not), with the property that the minimal isometry group acting on the universal

\footnotetext{
*Received December 11, 2008; accepted for publication October 9, 2009.

${ }^{\dagger}$ Department of Mathematics, Cornell University, Ithaca, NY 14853, USA (cao@math.cornell. edu). Research partially supported by the Jeffrey Sean Lehman Fund from Cornell University.

${ }^{\ddagger}$ Department of Mathematics, Cornell University, Ithaca, NY 14853, USA (lsc@math.cornell.edu). Research partially supported by NSF grant no. \# DMS 0603886.
} 
cover is unimodular. Let $g^{b}(t), t \in\left[0, T_{b}\right)$ be the maximal solution of the positive cross curvature flow $(+\mathrm{XCF})$ with initial metric $g_{0}$. Let $d(t)$ denote the distance function on $\left(M, g^{b}(t)\right)$. Assume that $g_{0}$ is generic among all locally homogeneous metrics on $M$. Then

- either $T_{b}=\infty$ and $g^{b}(t)=e^{\lambda t} g_{0}$ for some $\lambda \in \mathbb{R}$ (i.e., the cross curvature tensor of $g_{0}$ is equal to $\lambda g_{0}$ ),

- or $T_{b}<\infty$ and there exists a function $r(t):\left[0, T_{b}\right) \rightarrow(0, \infty)$ such that the metric spaces $(M, r(t) d(t))$ converge uniformly to a sub-Riemannian metric space $(M, d(T))$ whose tangent cone at any $m \in M$ is the Heisenberg group $\mathbb{H}_{3}$ equipped with its natural sub-Riemannian metric.

For a closed 3-dimensional Riemannian manifold $\left(M, g_{0}\right)$ that is locally homogeneous, there are 9 possibilities for the universal cover. They can be labelled by the minimal isometry group that acts transitively:

(a) $H(3)(H(n)$ denotes the isometry group of hyperbolic $n$-space); $\mathrm{SO}(3) \times \mathbb{R}$; $H(2) \times \mathbb{R}$

(b) $\mathbb{R}^{3} ; \mathrm{SU}(2) ; \mathrm{SL}(2, \mathbb{R})$; Heisenberg; $E(1,1)=\mathrm{Sol}$ (the group of isometry of plane with a flat Lorentz metric); $E(2)$ (the group of isometries of the Euclidean plane). This is called the Bianchi case in [IJ92].

In this paper, we prove Theorem 1.1 in all cases except for manifolds covered by

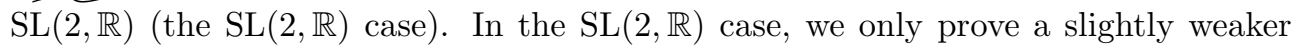
result (see Theorem 6.12). To obtain a proof of Theorem 1.1 in the $\operatorname{SL}(2, \mathbb{R})$ case, one actually needs some additional information. This additional information is obtained in [CGSC09] by using a different type of argument. See the comment at the end of the proof of Theorem 6.12 .

REMARK 1.1. The first case only occurs for homogeneous 3-manifolds covered by $\mathbb{R}^{3}, \mathbb{H}^{3}, \mathbb{S}^{2} \times \mathbb{R}$ and $\mathbb{H}^{2} \times \mathbb{R}$. Moreover, in those cases, $g^{b}(t)=e^{\lambda t} g_{0}$ for any homogeneous $g_{0}$.

We find it quite striking that the asymptotic behavior of $(+\mathrm{XCF})$, i.e., the backward behavior of $(-\mathrm{XCF})$, is essentially the same in all cases, for generic homogeneous metrics. In a companion paper [CSC09], we show that the same "universal" behavior holds for the backward Ricci flow on homogeneous 3-manifolds. This contrast with the very different behavior observed in the forward direction. See [IJ92, CNSC08] and the various more precise statements given below.

1.2. The cross curvature tensor on 3-manifolds. On a 3-dimensional Riemannian manifold $(M, g)$, let $R c$ be the Ricci tensor and $R$ be the scalar curvature. The Einstein tensor is defined by $E=R c-\frac{1}{2} R g$. Its local components are $E_{i j}=R_{i j}-\frac{1}{2} R g_{i j}$. Raising the indices, define $P^{i j} \stackrel{\frac{1}{2}}{=} g^{i k} g^{j l} R_{k l}-\frac{1}{2} R g^{i j}$, where $g^{i j}$ is the inverse of $g_{i j}$. Let $V_{i j}$ be the inverse of $P^{i j}$ (if it exists). The cross curvature tensor is (see [CH04])

$$
h_{i j}=\left(\frac{\operatorname{det} P^{k l}}{\operatorname{det} g^{k l}}\right) V_{i j} .
$$

Assume that computations are done in an orthonormal frame where the Ricci tensor, and thus also the cross curvature tensor, is diagonal. If the principal sectional curvatures are $k_{1}, k_{2}, k_{3}\left(k_{i}=K_{j k j k}\right.$, circularly) so that $R_{i i}=k_{j}+k_{l}$, circularly, then

$$
h_{i i}=k_{j} k_{l} .
$$

Notice that this definition works even when some of the sectional curvatures vanish. 
1.3. The cross curvature flows. In [CH04], Chow and Hamilton define the cross curvature flow on 3-manifolds starting from a metric with either positive sectional curvature or negative sectional curvature. More precisely, if $\epsilon= \pm 1$ is the sectional curvature sign (assumed to be well defined) of the metric $g_{0}$, the cross curvature flow starting from $g_{0}$ is the solution of

$$
\left\{\begin{array}{l}
\frac{\partial}{\partial t} g=-2 \epsilon h \\
g(0)=g_{0}
\end{array}\right.
$$

In these circunstances, the local existence of the flow was proved in [Buc06].

Locally homogeneous manifolds seldom have sectional curvatures that are all of the same sign. In dimension 3, positive sectional curvature is only possible on locally homogeneous manifolds covered by the sphere SU(2). Negative sectional curvature occurs only on hyperbolic 3-manifolds. All other locally homogeneous closed Riemannian 3-manifolds are either flat or have some positive sectional curvature [Mil76, Theorem 1.6]. Thus the definition above is not really practical for our purpose as far as a choice of sign is concerned and it is natural to investigate both the positive and the negative cross curvature flows defined by

$(+\mathrm{XCF}) \quad\left\{\begin{array}{l}\frac{\partial}{\partial t} g=2 h \\ g(0)=g_{0} .\end{array}\right.$

and

$$
\left\{\begin{array}{l}
\frac{\partial}{\partial t} g=-2 h \\
g(0)=g_{0}
\end{array}\right.
$$

In fact, starting from a initial metric $g_{0}$ on a locally homogeneous 3-manifold, let $g^{f}(t), t \in\left[0, T_{f}\right)$ be the maximal forward solution of the (-XCF), and $g^{b}(t), t \in\left[0, T_{b}\right)$ be the maximal forward solution of the $(+\mathrm{XCF})$. Now, for $t \in I=\left(-T_{b}, T_{f}\right)$, set

$$
g(t)=\left\{\begin{array}{cc}
g^{f}(t) & \text { for } t \in\left[0, T_{f}\right) \\
g^{b}(-t) & \text { for } t \in\left(-T_{b}, 0\right] .
\end{array}\right.
$$

It is easy to see that, by construction, $g(t), t \in I$, is a maximal solution of (-XCF) passing through $g_{0}$ at time $t=0$. One of the goals of our study is to describe the behavior of these maximal solutions in the forward and backward directions. The forward direction is treated in [CNSC08] and the present paper is devoted to the backward direction which, of course, is the same as the forward direction for $(+\mathrm{XCF})$.

1.4. Normalizations. Let $g(t), t \in I$, be a maximal solution of the (-XCF). By renormalization of $g(t)$, we mean a family $\widetilde{g}(\widetilde{t}), \widetilde{t} \in \widetilde{I}$, obtained by a change of scale in space and a change of time, that is

$$
\widetilde{g}(\widetilde{t})=\psi(t) g(t), \widetilde{t}=\int_{0}^{t} \psi^{2}(s) d s .
$$

Set $\widetilde{\psi}(\widetilde{t})=\psi(t)$, then we have

$$
\frac{\partial \widetilde{g}}{\partial \widetilde{t}}=-2 \widetilde{h}+\left(\frac{d}{d \widetilde{t}} \ln \widetilde{\psi}\right) \widetilde{g},
$$

where $\widetilde{h}$ is the cross curvature tensor of $\widetilde{g}$. 
On compact manifolds, it is customary to take $\frac{d}{d t} \ln \psi=\frac{2}{3} \bar{h}$, where $\bar{h}=\frac{\int \operatorname{tr}(h) d \mu}{\int d \mu}$ is the average of the trace of the cross curvature because the volume of the metric $\widetilde{g}$ is then constant. In this paper, we will consider some different normalizations, for instance, keeping the diameter constant. Moreover, we will not worry about the time change associated above with a re-scaling by $\psi$. Given a solution $g$ of $(-\mathrm{XCF})$, we will be interested in finding re-scaling $\bar{g}(t)=\phi(t) g(t)$ such that the asymptotic behavior of the metric space $(M, \bar{g}(t))$ is described by a model having the largest possible dimension (i.e., minimum collapse). Although making this precise could possibly involve some difficulties in general, in our specific examples, what it means will be quite obvious.

1.5. Convergence of metric spaces. We refer the reader to [BBI01] for an introduction to and more details on the notions discussed briefly in this section. We start with the most basic (and naive) notion of convergence: the uniform convergence of a family of metric spaces $\left(X, d_{t}\right), t \in(0, T)$, towards a metric space $\left(X, d_{T}\right)$ when all metric structures are defined on the same topological space $X$. By definition, this uniform convergence (which we will encounter frequently below), is simply the convergence of the functions $d_{t}$ to $d_{T}$ on $X \times X$, uniformly on compact sets, as $t$ tends to $T$.

ExAmple 1.1. Let $g_{t}, t \in(0, T]$, be Riemannian metrics on a manifold $M$, equipped with an auxiliary Riemannian metric $g_{0}$. Let $d_{t}$ be the corresponding distance functions on $M$. Assume that there is a Riemannian metric $g_{T}$ on $M$ such that for any compact $K \subset M$,

$$
\lim _{t \rightarrow T} \max _{x \in K} \max _{u \in T_{x}: g_{0}(u, u) \leq 1}\left|g_{t}(u, u)-g_{T}(u, u)\right|=0 .
$$

Then the distance $d_{t}$ converge uniformly on compact sets to $d_{T}$.

The Lipschitz distance between two metric spaces is the infimum of $\ln \operatorname{dil}(f)+$ $\ln \operatorname{dil}\left(f^{-1}\right)$ where $f$ is a bi-lipschitz homeomorphism between $X$ and $Y$ and

$$
\operatorname{dil}(f)=\sup _{x, x^{\prime} \in X}\left\{d_{Y}\left(f(x), f\left(x^{\prime}\right)\right) / d_{X}\left(x, x^{\prime}\right)\right\} .
$$

ExAmple 1.2. Let $M$ and $g_{t}, t \in[0, T]$ be as in Example 1.1. If (1.2) holds and $M$ is compact then the Lipschitz distance between $\left(M, d_{t}\right)$ and $\left(M, d_{T}\right)$ tends to zero. If $M$ is not compact, this is not necessarily the case because Lipschitz convergence implies some global control. However, if one assumes instead that

$$
\lim _{t \rightarrow T} \max _{x \in M} \max _{u \in T_{x}: g_{0}(u, u) \leq 1}\left|g_{t}(u, u)-g_{T}(u, u)\right|=0
$$

then the Lipschitz distance between $\left(M, d_{t}\right)$ and $\left(M, d_{T}\right)$ tends to zero.

Recall that for two subsets $A, B$ of a metric space $Z$,

$$
d_{H}^{Z}(A, B)=\inf \left\{\epsilon>0: A \subset B_{\epsilon} \text { and } B \subset A_{\epsilon}\right\}
$$

where $A_{\epsilon}$ is the $\epsilon$-neighborhood of $A$ in $Z$. The Hausdorff distance between two metric spaces $X, Y$ is the infimum of the numbers $d_{H}^{Z}(f(X), g(Y))$ for all metric spaces $Z$ and all isometric embeddings $f, g$ of $X, Y$ into $Z$. Finally, a sequence of pointed metric spaces $\left(X_{n}, p_{n}\right)$ converges in the Gromov-Hausdorff sense to a pointed metric space 
$(X, p)$ if, for every $r, \epsilon>0$, there is a map $f: B_{X_{n}}\left(p_{n}, r\right) \rightarrow X$ (not necessarily continuous) such that $f\left(p_{n}\right)=p, \operatorname{dis}(f)<\epsilon, B_{X}(p, r-\epsilon) \subset\left[f\left(B_{X_{n}}\left(, p_{n}, r\right)\right)\right]_{\epsilon}$. Here, if $f$ is a map from $X$ to $Y$, $\operatorname{dis}(f)=\sup _{x, x^{\prime} \in X}\left|d_{Y}\left(f(x), f\left(x^{\prime}\right)\right)-d_{X}\left(x, x^{\prime}\right)\right|$. For length spaces (and we will deal only with length spaces), this is equivalent to say that the balls $B_{X_{n}}\left(p_{n}, r\right)$ converge in the Gromov-Hausdorff sense to $B(p, r)$, for all each $r>0$.

EXAmple 1.3. Convergence in the Gromov-Hausdorff sense allows for dimensional collapse. For instance, the pointed cylinder $\left(\mathbb{R} \times S^{1},(0,0), d_{t}\right), d_{t}$ being the distance associated with $g_{t}=(d x)^{2}+t^{-1}(d \theta)^{2}$ converges in the Gromov-Hausdorff sense as $t$ tends to infinity to $(\mathbb{R}, 0)$ equipped with its usual metric.

Note that the metric spaces $\left(\mathbb{R}^{2},(0,0), d_{t}\right), d_{t}$ associated with $g_{t}=(d x)^{2}+$ $t^{-1}(d y)^{2}$, are all isometric and thus indistinguishable in terms of the Lipschitz or Gromov-Hausdorff distances.

EXAmple 1.4. [Tangent cones] Given a pointed metric space $(X, d, p)$, we call tangent cone at $p$, any pointed metric space $\left(X_{0}, d_{0}, p_{0}\right)$ which appears as a GromovHausdorff limit of (a subsequence of) the family of pointed metric spaces $(X, t d, p), t$ tending to 0 . For any pointed Riemannian $n$-manifold $(M, g, p)$, the tangent cone at $p$ exists, is unique, and equals the Euclidean $n$-space.

1.6. Locally homogeneous 3-manifolds. By classical arguments, the study of the Ricci or cross curvature flow on a locally homogeneous manifold reduces essentially to the study of the same flow on the universal cover. In dimension 3 there are 9 possibilities for the universal cover, four of which are essentially trivial as far as the cross curvature flow is concerned. These four easy cases are : $\mathbb{R}^{3}$ (flat metrics), $\mathbb{H}^{3}$ (hyperbolic metric), $\mathbb{S}^{2} \times \mathbb{R}$ and $\mathbb{H}^{2} \times \mathbb{R}$. See [CNSC08]. In the remaining 5 cases the universal cover is itself a group that act transitively on the manifold. This paper focusses on these five cases which are: $\mathrm{SU}(2), \widetilde{\mathrm{SL}(2, \mathbb{R})}$; Heisenberg; $E(1,1)=$ Sol (the group of isometry of plane with a flat Lorentz metric); $\widetilde{E(2)}$ (the universal cover of the group of isometries of the Euclidean plane). See [IJ92] or [CNSC08] for a more detailed discussion.

Assume that $\mathfrak{g}$ is a 3 -dimensional real Lie unimodular algebra equipped with an oriented Euclidean structure. According to J. Milnor, [Mil76], there exists a (positively oriented) orthonormal basis $\left(e_{1}, e_{2}, e_{3}\right)$ and reals $\lambda_{1}, \lambda_{2}, \lambda_{3}$ such that the bracket operation of the Lie algebra has the form

$$
\left[e_{i}, e_{j}\right]=\lambda_{k} e_{k} \quad(\text { circularly in } i, j, k) .
$$

Milnor shows that such a basis diagonalizes the Ricci tensor and thus also the cross curvature tensor. If $f_{i}=a_{j} a_{k} e_{i}$ with nonzero $a_{i}, a_{j}, a_{k} \in \mathbb{R}$, then $\left[f_{i}, f_{j}\right]=\lambda_{k} a_{k}^{2} f_{k}$ (circularly in $i, j, k$ ). Using the choice of orientation, we may assume that at most one of the $\lambda_{i}$ is negative and then, the Lie algebra structure is entirely determined by the signs (in $\{-1,0,+1\}$ ) of $\lambda_{1}, \lambda_{2}, \lambda_{3}$ as follows:

$$
\begin{array}{llll}
+ & + & + & \mathrm{SU}(2) \\
+ & + & - & \mathrm{SL}(2, \mathbb{R}) \\
+ & + & 0 & E(2)(\text { Euclidean motions in } 2 D) \\
+ & 0 & - & E(1,1)(\text { also called Sol) } \\
+ & 0 & 0 & \text { Heisenberg group } \\
0 & 0 & 0 & \mathbb{R}^{3}
\end{array}
$$


In each case, let $\epsilon=\left(\epsilon_{1}, \epsilon_{2}, \epsilon_{3}\right) \in\{-1,0,+1\}^{3}$ be the corresponding choice of signs. Then, given $\epsilon$ and an Euclidean metric $g_{0}$ on the corresponding Lie algebra, we can choose a basis $f_{1}, f_{2}, f_{3}$ (with $f_{i}$ collinear to $e_{i}$ above) such that

$$
\left[f_{i}, f_{j}\right]=2 \epsilon_{k} f_{k} \quad(\text { circularly in } i, j, k) .
$$

We call $\left(f_{i}\right)_{1}^{3}$ a Milnor frame for $g_{0}$. The metric, the Ricci tensor and the cross curvature tensor are all diagonalized in this basis and this property is obviously maintained throughout either the Ricci flow or cross curvature flow. If we let $\left(f^{i}\right)_{1}^{3}$ be the dual frame of $\left(f_{i}\right)_{1}^{3}$, the metric $g_{0}$ has the form

$$
g_{0}=A_{0} f^{1} \otimes f^{1}+B_{0} f^{2} \otimes f^{2}+C_{0} f^{3} \otimes f^{3} .
$$

Assuming existence of the flow $g(t)$ starting from $g_{0}$, under either the Ricci flow or the cross curvature flow (positive or negative), the original frame $\left(f_{i}\right)_{1}^{3}$ stays a Milnor frame for $g(t)$ along the flow and $g(t)$ has the form

$$
g(t)=A(t) f^{1} \otimes f^{1}+B(t) f^{2} \otimes f^{2}+C(t) f^{3} \otimes f^{3} .
$$

It follows that these flows reduce to ODEs in $(A, B, C)$. Given a flow, the explicit form of the ODE depends on the underlying Lie algebra structure. With the help of the curvature computations done by Milnor in [Mil76], one can find the explicit form of the equations for each Lie algebra structure. The Ricci flow case was treated in [IJ92]. The computations of the ODEs corresponding to the cross curvature flow are presented in [CNSC08] and will be used below to study the asymptotic behavior of $(+\mathrm{XCF})$.

1.7. Sub-Riemannian Geometry. The notion of sub-Riemannian geometry can be described from several equivalent but different viewpoints. The simplest is perhaps to start with a family of smooth vector fields $\mathcal{X}=\left\{X_{i}, i \in\{1, \ldots, k\}\right\}$ on a manifold $M$ with the property that these fields, together with their brackets of all orders, span the tangent space at each point of $M$. This is often called Hörmander's condition (Hörmander proved that the associated sum of squares $L=\sum X_{i}^{2}$ is hypoellitic). Given a family $\mathcal{X}$, one defines a distance on $M$ as follows. A vector $u$ in the tangent space $T_{x}$ at $x$ is subunit if $u=\sum_{1}^{k} a_{i} X_{i}(x)$ with $\sum\left|a_{i}\right|^{2} \leq 1$. An absolutely continuous curve $\gamma:[0, T] \rightarrow M$ is subunit if $\dot{\gamma}(t)$ is subunit for each $t \in[0, T]$ (in particular, this means that $\dot{\gamma}(t)$ belongs to the span of $\mathcal{X}$ at $\gamma(t)$, i.e., is horizontal). The distance $d_{\mathcal{X}}(x, y)$ is the infimum of $T$ such that there exists an absolutely continuous subunit curve $\gamma:[0, T] \rightarrow M$ with $\gamma(0)=x$ and $\gamma(T)=y$.

Another equivalent definition starts with a distribution $H$, that is to say, a subbundle of the tangent bundle, together with a fiber inner product on this sub-bundle. This easily leads to the notion of length of a horizontal curve (i.e., a curve that stays tangent to the sub-bundle). In this case, the Hörmander condition is expressed using a local frame for $H$.

A third equivalent definition is based on the choice of a symmetric non-negative $(0,2)$-tensor $Q$ (a possibly degenerate inner product on the co-tangent bundle). This defines a sub-bundle of the tangent bundle by setting

$$
H_{x}=\left\{u \in T_{x}: \sup _{\alpha: Q(\alpha, \alpha) \leq 1} \alpha(u)<\infty\right\}
$$

and induces an inner product on $H_{x}$ in the obvious way. Again, Hörmander's condition can be expressed using a local frame for $H$. The link between the first presentation 
and the third is simply that, given a Hörmander family $\mathcal{X}$,

$$
Q(\alpha, \alpha)=\sum_{1}^{k}\left|\alpha\left(X_{i}\right)\right|^{2} .
$$

The most basic result of sub-Riemannian geometry is that, assuming Hörmander's condition, the associated sub-Riemannian distance defines the original topology of the manifold $M$. See Chow's theorem in [Mon02, Ch. 2]. More generally, we refer the reader to [Mon02] for a detailed introduction to sub-Riemannian geometry.

Most relevant to the present paper is the fact that sub-Riemannian metrics can easily appear as limit of Riemannian metrics as explain in the following example.

EXAMPLE 1.5. Let $M$ be a manifold, equipped with a family of Riemannian metric $g_{t}, t \in[0, T)$ (we will use $g_{0}$ as a reference metric here). For each $t \in[0, T$ ), we let $d_{t}$ be the corresponding distance function. Each $g_{t}$ induces a symmetric positive definite $(0,2)$-tensor $Q_{t}$. Now, the existence of a Riemannian metric $g_{T}$ such that

$$
\lim _{t \rightarrow T} \max _{x \in K} \max _{\substack{u \in T_{x}: \leq \\ g_{0}(u, u) \leq 1}}\left|g_{t}(u, u)-g_{T}(u, u)\right|=0
$$

is obviously equivalent to the the existence of a symmetric positive definite $(0,2)$ tensor $Q_{T}$ such that

$$
\lim _{t \rightarrow T} \max _{x \in K} \max _{\substack{u \in T_{x}: \\ Q_{0}(u, u) \leq 1}}\left|Q_{t}(u, u)-Q_{T}(u, u)\right|=0 .
$$

However, in general, it is well possible that there exists a symmetric non-negative $(0,2)$-tensor $Q_{T}$ such that

$$
\lim _{t \rightarrow T} \max _{x \in K} \max _{\substack{u \in T_{x}^{\prime} \\ Q_{0}(u, u) \leq 1}}\left|Q_{t}(u, u)-Q_{T}(u, u)\right|=0
$$

even if the metrics $g_{t}$ do not have a well defined finite limit. In that case, if the limiting $(0,2)$-tensor $Q_{T}$ turns out to satisfy Hörmander's condition then the metric space $\left(M, d_{t}\right)$ converges uniformly on compact sets to the sub-Riemannian metric space $\left(M, d_{T}\right)$ where $d_{T}$ is the sub-Riemannian distance function associated with $Q_{T}$. See, e.g., [JSC87].

The case of left-invariant sub-Riemannian structures on Lie groups is somewhat simpler than the general case and extremely natural. Recall that the Lie algebra $\mathfrak{g}$ of a connected Lie group $G$ can be identify with the space of left-invariant vector fields equipped with the bracket operation. A left-invariant sub-Riemannian structure on $G$ is simply a family $\mathcal{X}=\left\{X_{1}, \ldots, X_{k}\right\}$ of left-invariant vector fields which generates the Lie algebra. The associated left-invariant quadratic form on the cotangent bundle is given by (1.8). We briefly illustrate this case by examples of sub-Riemannian geometries on the Heisenberg group and on $\mathrm{SU}(2)$.

EXAMPLE 1.6. The Heisenberg group $\mathbb{H}_{3}$ is $\mathbb{R}^{3}$ equipped with the multiplication

$$
(x, y, z) \cdot\left(x^{\prime}, y^{\prime}, z^{\prime}\right)=\left(x+x^{\prime}, y+y^{\prime}, z+z^{\prime}+\frac{1}{2}\left(x y^{\prime}-y x^{\prime}\right)\right) .
$$

It is easy to see that the left-invariant vector fields equal to $d / d x, d / d y$ and $d / d z$ at $(0,0,0)$ are

$$
X=d / d x-(y / 2) d / d z, Y=d / d y+(x / 2) d / d z \text { and } Z=d / d z .
$$


Moreover, $Z=[X, Y]$ and $[X, Z]=[Y, Z]=0$. The "canonical" sub-Riemannian structure on $\mathbb{H}_{3}$ is associated with the (minimal) Hörmander family $\{X, Y\}$. This structure is particularly adapted to $\mathbb{H}_{3}$ because it is homogeneous with respect to the natural dilations $\delta_{s}(p)=\left(s x, s y, s^{2} z\right), p=(x, y, z)$, that commutes with the group law. Namely, if $d\left(p, p^{\prime}\right)$ is the sub-Riemannian distance associated with the family $\{X, Y\}$, we have

$$
d\left(\delta_{s}(p), \delta_{s}\left(p^{\prime}\right)\right)=s d\left(p, p^{\prime}\right), \quad p, p^{\prime} \in \mathbb{H}_{3} .
$$

No left-invariant Riemannian metrics can have this property. There is an exact expression for the sub-Riemannian distance on $\mathbb{H}_{3}$ (this is one of the very few cases of sub-Riemannian geometry where such an exact formula exists). To connect with the notation introduced in our discussion of Milnor frame on 3-dimensional Lie groups, observe that $f_{1}=Z / 2, f_{2}=X, f_{3}=Y$ is a Milnor frame for the left-invariant metric on $\mathbb{H}_{3}$ given at the origin by $g_{0}=d x^{2}+d y^{2}+d z^{2}$. The sub-Riemannian structure on $\mathbb{H}_{3}$ discussed above can be described as

$$
Q=f_{2} \otimes f_{2}+f_{3} \otimes f_{3} .
$$

Note that this can be viewed as the limit of any family of Riemannian metrics

$$
g_{t}=A(t) f^{1} \otimes f^{1}+B(t) f^{2} \otimes f^{2}+C(t) f^{3} \otimes f^{3}, \quad t \in[0, T)
$$

such that $\lim _{T} B=\lim _{T} C=1$ and $\lim _{T} A=\infty$. Indeed, in this case,

$$
Q_{t}=A(t)^{-1} f_{1} \otimes f_{1}+B(t)^{-1} f_{2} \otimes f_{2}+C(t)^{-1} f_{3} \otimes f_{3}, \quad t \in[0, T)
$$

which obviously tends to $Q$ (uniformly!). Note that the existence of the dilations $\delta_{s}, s>0$, imply immediately that the tangent cone at the identity element $e$ of the pointed sub-Riemannian metric space $\left(\mathbb{H}_{3}, Q, e\right)$ is that space itself.

EXAMPLE 1.7. The group $\mathrm{SU}(2)$ is the group of matrices

$$
\left\{\left(\begin{array}{cc}
a & b \\
-\bar{b} & \bar{a}
\end{array}\right): a, b \in \mathbb{C},|a|^{2}+|b|^{2}=1\right\}
$$

which can also be identified with the 3 -sphere $\mathbb{S}^{3}$. Its Lie algebra can be identified with

$$
\mathfrak{s u}(2)=\left\{\left(\begin{array}{cc}
i \alpha & \beta \\
-\bar{\beta} & i \alpha
\end{array}\right): \alpha \in \mathbb{R}, \beta \in \mathbb{C}\right\} .
$$

Let $g_{0}$ be any left-invariant Riemannian metric on $\mathrm{SU}(2)$ and $f_{1}, f_{2}, f_{3}$ be a Milnor frame as defined earlier. Computation of the sectional curvatures (see below) show that $\left(f_{1}, f_{2}, f_{3}\right)$ is always an orthonormal frame for the standard round sphere metric on $\mathrm{SU}(2) \simeq \mathbb{S}^{3}$. Since $\left[f_{i}, f_{j}\right]=2 f_{k}$ circularly, we can pick any two of these vectors, say $f_{2}, f_{3}$, and consider the sub-Riemannian metrics

$$
Q_{b, c}=b f_{2} \otimes f_{2}+c f_{3} \otimes f_{3}
$$

where $b, c$ are fixed positive constants. These obviously appears as limits of Riemannian metrics as in the case of the Heisenberg group discussed above. The tangent cone of $\mathrm{SU}(2)$ equipped with one of this sub-Riemannian metric is, at any fixed point, the Heisenberg group equipped with its canonical sub-Riemannian structure discussed above. For a discussion of this example and relation to the Hopf fibration, see [Mon02]. 
2. The cross curvature flow on the Heisenberg group. Given a metric $g_{0}$ on the Heisenberg group (or on a 3 manifold of Heisenberg type), we fix a Milnor frame $\left\{f_{i}\right\}_{1}^{3}$ such that

$$
\left[f_{2}, f_{3}\right]=2 f_{1}, \quad\left[f_{3}, f_{1}\right]=0, \quad\left[f_{1}, f_{2}\right]=0
$$

and (1.6)-(1.7) hold. Using [Mil76], the sectional curvatures are:

$$
K\left(f_{2} \wedge f_{3}\right)=-\frac{3 A}{B C}, \quad K\left(f_{3} \wedge f_{1}\right)=K\left(f_{1} \wedge f_{2}\right)=\frac{A}{B C} .
$$

and the scalar curvature is $R=-2 A / B C$. The ODE for $(+\mathrm{XCF})$ is given by

$$
\left\{\begin{array}{l}
\frac{d A}{d t}=2 \frac{A^{3}}{B^{2} C^{2}}, \\
\frac{d B}{d t}=-6 \frac{A^{2}}{B C^{2}} \\
\frac{d C}{d t}=-6 \frac{A^{2}}{B^{2} C}
\end{array}\right.
$$

This case is very simple and admits a completely explicit solution. Since the computations for $(+\mathrm{XCF})$ are essentially the same as for $(-\mathrm{XCF})$, we refer the reader to [CNSC08] for details and simply write down the explicit maximal solution of (-XCF) passing through $g_{0}$ at $t=0$.

Theorem 2.1. Given $A_{0}, B_{0}, C_{0}>0$ and $R_{0}=2 A_{0} /\left(B_{0} C_{0}\right)$, set

$$
T_{0}=T_{b}=1 /\left(7 R_{0}^{2}\right) .
$$

The maximal solution of $(-\mathrm{XCF})$ through $g_{0}$ at $t=0$ is defined on $\left(-T_{0}, \infty\right)$ and given by

$$
\left\{\begin{array}{l}
A(t)=A_{0}\left(1+t / T_{0}\right)^{-\frac{1}{14}} \\
B(t)=B_{0}\left(1+t / T_{0}\right)^{\frac{3}{14}} \\
C(t)=C_{0}\left(1+t / T_{0}\right)^{\frac{3}{14}}
\end{array}\right.
$$

The sectional curvatures are given by

$$
-\frac{1}{3} K\left(f_{2} \wedge f_{3}\right)=K\left(f_{1} \wedge f_{2}\right)=K\left(f_{3} \wedge f_{1}\right)=\frac{A_{0}}{B_{0} C_{0}}\left(1+t / T_{0}\right)^{-\frac{1}{2}}
$$

From the view point of the positive cross curvature flow $(+\mathrm{XCF})$, this theorem indicates that $(+\mathrm{XCF})$ on the Heisenberg group develops a singularity at the finite time $T_{0}=B_{0}^{2} C_{0}^{2} /\left(28 A_{0}^{2}\right)$. This singularity is of a type that is different from the singularities usually observed in geometric flows which are dimensional collapses such as pancake and cigar degeneracies.

Theorem 2.1 clearly indicates that it is natural to re-scale the metric $g(t)$ by a factor of $\psi(t)=\left(1+t / T_{0}\right)^{-3 / 14}$ (we will ignore the corresponding change of time $\widetilde{t}=\int_{0}^{t} \psi(s)^{2} d s$ but note that the backward blow-up time stays finite anyhow). Accordingly, we set

$$
\bar{g}(t)=\left(1+t / T_{0}\right)^{-3 / 14} g(t)
$$


THEOREM 2.2. Let $M$ be a complete locally homogeneous 3-manifolds of Heisenberg type with initial homogeneous metric $g_{0}$ and associated Milnor frame $\left(f_{1}, f_{2}, f_{3}\right)$. Let $\bar{g}$ be as defined above.

1. If $M$ is compact, as t tends to infinity, the metric space $(M, g(t))$ converges in the Gromov-Hausdorff sense to $\mathbb{R}^{2}$ with a flat metric (the sectional curvature tends to 0 ). If $M=\mathbb{H}_{3}$, as $t$ tends to infinity, the pointed metric space $\left(\mathbb{H}_{3}, e, g(t)\right)$ converges in the Gromov-Hausdorff sense to $\mathbb{R}^{3}$ with a flat metric.

2. As $t$ tends to $-T_{0}$, the metric space $(M, \bar{g}(t))$ converges uniformly to the sub-Riemannian metric space $\left(M, B_{0}^{-1} f_{2} \otimes f_{2}+C_{0}^{-1} f_{3} \otimes f_{3}\right)$.

REMARK 2.1. In the first statement, the direction that collapses is that of the center, i.e., $f_{1}$. It follows that to have Gromov-Hausdorff convergence to a flat $\mathbb{R}^{2}$, it suffices to assume that $M$ is of the form $\mathbb{H}_{3} / \Gamma$ where $\Gamma$ is a discrete subgroup of $\mathbb{H}_{3}$ with non-trivial intersection with the center. If $\Gamma$ has trivial intersection with the center then $M=\mathbb{H}_{3} / \Gamma$ is not compact and converges in the pointed Gromov-Hausdorff sense to a flat $\mathbb{R}^{3}$.

REMARK 2.2. In the second statement, the identity map Id is, in fact, a biLipschitz map between $(M, \bar{g}(t))$ and $\left(M, B_{0}^{-1} f_{2} \otimes f_{2}+C_{0}^{-1} f_{3} \otimes f_{3}\right)$ and dil(Id) defined at (1.3) tends to 0 as $t$ tends to $-T_{0}$.

REMARK 2.3. Let $e_{i}^{t}$ be the unit vector for the metric $g(t)$ positively collinear to $f_{i}$. It is useful to look at the evolution of the Lie algebra structure viewed from the perspective of the metric $g(t)$. Namely, we have

$$
\left[e_{2}^{t}, e_{3}^{t}\right]=\sqrt{A(t) / B(t) C(t)} e_{1}^{t}, \quad\left[e_{1}^{t}, e_{2}^{t}\right]=\left[e_{3}^{t}, e_{1}^{t}\right]=0 .
$$

As $t$ tends to $\infty, A(t) / B(t) C(t)$ tends to 0 and the non-trivial nilpotent structure converges to the trivial abelian structure on $\mathbb{R}^{3}$. This is geometrically significant here since the exponential map yields global coordinates.

3. The cross curvature flow on $\mathbf{S U}(2)$. Given a metric $g_{0}$ on $S U(2)$, we fix a Milnor frame such that $\left[f_{i}, f_{j}\right]=2 f_{k}$. For any metric $g=A f^{1} \otimes f^{1}+B f^{2} \otimes f^{2}+$ $C f^{3} \otimes f^{3}$, the sectional curvatures are (see, e.g., [CK04, pg. 12]

$$
\begin{aligned}
& K\left(f_{2} \wedge f_{3}\right)=\frac{(B-C)^{2}}{A B C}-\frac{3 A}{B C}+\frac{2}{B}+\frac{2}{C}, \\
& K\left(f_{3} \wedge f_{1}\right)=\frac{(C-A)^{2}}{A B C}-\frac{3 B}{C A}+\frac{2}{A}+\frac{2}{C}, \\
& K\left(f_{1} \wedge f_{2}\right)=\frac{(A-B)^{2}}{A B C}-\frac{3 C}{A B}+\frac{2}{A}+\frac{2}{B} .
\end{aligned}
$$

Together with the results obtained in [CNSC08], the asymptotic behavior that will be proved in this section (see Theorem 3.8 below) give the following full description of the asymptotic behavior of the solution of the negative cross curvature flow passing through a metric $g_{0}$ at time 0 .

Theorem 3.1. Let $g(t)=A(t) f^{1} \otimes f^{1}+B(t) f^{2} \otimes f^{2}+C(t) f^{3} \otimes f^{3}, t \in\left(-T_{b}, T_{f}\right)$, be a maximal solution of the negative cross curvature flow (-XCF) on $\mathrm{SU}(2)$. Assume that $A_{0} \geq B_{0} \geq C_{0}$ and set

$$
\bar{g}(t)=\frac{B_{0}}{B(t)} g(t)
$$


- The time $T_{f}$ is finite. When $t \rightarrow T_{f}$, $(\mathrm{SU}(2), \bar{g}(t))$ converges uniformly to the round sphere $\mathbb{S}_{\sqrt{B_{0}}}^{3}$.

- If $A_{0}=B_{0}=C_{0}$, then $T_{b}=\infty, T_{f}=B_{0}^{2} / 4, B(t)=\sqrt{B_{0}^{2}-4 t}$ and $\bar{g}(t)=g_{0}$ (a round metric) for all $t \in\left(-\infty, T_{f}\right)$.

- If $A_{0}=B_{0}>C_{0}$ (the Berger sphere case), then $T_{b}=\infty$ and, as $t \rightarrow-\infty$, $(\mathrm{SU}(2), \bar{g}(t))$ converges in the Gromov-Hausdorff sense to a two-dimensional round sphere (the sectional curvatures of $\bar{g}(t)$ containing $f_{3}$ tends to 0 whereas the one corresponding to $f_{1} \wedge f_{2}$ tends to $\left.4 / B_{0}\right)$.

- If $A_{0}>B_{0} \geq C_{0}$ then $T_{b}$ is finite. As $t \rightarrow-T_{b}$, (SU(2), $\left.\bar{g}(t)\right)$ converges uniformly to a sub-Riemannian metric space (SU(2), bf $\left.f_{2} \otimes f_{2}+c f_{3} \otimes f_{3}\right)$ with $b=B_{0}^{-1}$ and $c \in\left[B_{0}^{-1}, \infty\right)$ (if $B_{0}>C_{0}$ then $b>c$ ).

Proof. Theorem 3 of [CNSC08] gives $T_{f} \in(0, \infty)$ and the asymptotic $A, B, C \sim$ $2 \sqrt{T-t}$ as $t$ tends to $T_{f}$. The first statement follows. The other statements are consequences of Theorem 3.8 below.

REMARK 3.1. If one think about the global behavior of the two-sided maximal flow lines of the cross curvature flow in a given Milnor frame on $\mathrm{SU}(2)$ in terms of the value taken at time $0,\left(A_{0}, B_{0}, C_{0}\right)$, in the first octant of $\mathbb{R}^{3}$, one should distinguish 6 regions, each corresponding to a strict order of the type $A>B>C$. These regions are preserved by the flow and separated by planes of the type $A=B$ corresponding to Berger sphere metrics. These planes are preserved by the flow. The intersection of these planes is the line $A=B=C$ corresponding to round metrics. This line is also preserved by the flow. In the forward direction, the flow lines all approach the line $A=B=C$, towards the point $(0,0,0)$. In the backward direction, in each of the 6 open regions, the largest component tends to infinity whereas the two smaller components have finite distinct limits. On the plane $A=B$, in the backward direction, $A=B$ tends to infinity and $C$ has a finite limit.

This section is devoted to the proof of the result that concern the behavior at $-T_{b}$. For the rest of this section, we only consider forward solutions of $(+\mathrm{XCF})$.

From the sectional curvatures given above, we easily obtain the ODEs corresponding to the positive cross curvature flow $(+\mathrm{XCF})$, namely,

$$
\left\{\begin{array}{l}
\frac{d A}{d t}=2 \frac{A Y Z}{(A B C)^{2}}, \\
\frac{d B}{d t}=2 \frac{B Z X}{(A B C)^{2}} \\
\frac{d C}{d t}=2 \frac{C X Y}{(A B C)^{2}}
\end{array}\right.
$$

where

$$
\begin{aligned}
& X=3 A^{2}-(B-C)^{2}-2 A B-2 A C, \\
& Y=3 B^{2}-(A-C)^{2}-2 A B-2 B C \\
& Z=3 C^{2}-(A-B)^{2}-2 B C-2 A C
\end{aligned}
$$


Recall from [CNSC08], that we have

$$
\begin{aligned}
& \frac{d \ln (A / B)}{d t}=\frac{8 Z}{(A B C)^{2}}(B-A)(A+B-C), \\
& \frac{d \ln (A / C)}{d t}=\frac{8 Y}{(A B C)^{2}}(A-C)(B-C-A), \\
& \frac{d \ln (B / C)}{d t}=\frac{8 X}{(A B C)^{2}}(B-C)(A-B-C),
\end{aligned}
$$

and

$$
\begin{aligned}
& \frac{d(A-B)}{d t}=\frac{2 Z}{(A B C)^{2}}(B-A)\left[A^{2}+A(6 B-2 C)+(B-C)^{2}\right] \\
& \frac{d(B-C)}{d t}=\frac{2 X}{(A B C)^{2}}(C-B)\left[(A-B-C)^{2}+4 B C\right], \\
& \frac{d(A-C)}{d t}=\frac{2 Y}{(A B C)^{2}}(C-A)\left((A-B)^{2}+6 A C-2 B C+C^{2}\right) .
\end{aligned}
$$

Without loss of generality we may assume that $A_{0} \geq B_{0} \geq C_{0}$ and it is easy to see from (3.5) and (3.6) that $A \geq B \geq C$ is preserved along the flow. As a consequence, we have

$$
\begin{aligned}
& Y=(B-A)(A+B+2 B-2 C)-C^{2} \leq-C^{2}<0, \\
& Z=-(A-B)^{2}+3 C^{2}-2 A C-2 B C \leq-C^{2}<0,
\end{aligned}
$$

and this implies the following Lemma.

Lemma 3.2. Assume that $A_{0} \geq B_{0} \geq C_{0}$. Then $A, A / B, A / C, A-B$ and $A-C$ are all nondecreasing along $(+\mathrm{XCF})$.

We will consider three cases.

Case 1: $A_{0}=B_{0}=C_{0}$.

In this case, which is the round sphere, $A(t)=B(t)=C(t)=\sqrt{A_{0}^{2}+4 t}$, and the solution exists for all time $t$.

Case 2: $A_{0}=B_{0}>C_{0}$.

In this case, $A=B>C$ as long as the solution exist. The equations simplify to

$$
\left\{\begin{array}{l}
\frac{d A}{d t}=2 \frac{4 A C-3 C^{2}}{A^{3}}, \\
\frac{d C}{d t}=2 \frac{C^{3}}{A^{4}} .
\end{array}\right.
$$

Clearly, $A$ and $C$ are increasing. Since $A \frac{d}{d t} A<8$, the solution exists for all time $t \in[0, \infty)$.

Lemma 3.3. Assume $A_{0}=B_{0}>C_{0}$. Then

$$
\lim _{t \rightarrow \infty} A(t)=\infty \text { and } \lim _{t \rightarrow \infty} C(t)=C_{\infty}<\infty .
$$

Moreover, as $t \rightarrow \infty$,

$$
A(t) \sim\left(24 C_{\infty} t\right)^{1 / 3} \text { and } C(t)-C_{\infty} \sim-2^{-3} 3^{-1 / 3} C_{\infty}^{5 / 3} t^{-1 / 3} .
$$


Proof. Assume that $\lim _{\infty} A=\eta<\infty$. Since $A / C$ is nondecreasing, we have

$$
\frac{d}{d t} \ln (A / C)=\frac{8 C(A-C)}{A^{4}} \geq \eta>0
$$

and thus $\ln (A / C)>\eta t$. This is a contradiction.

Now assume that $\lim _{\infty} C=\infty$. We first show that

$$
\lim _{\infty} A / C=\infty
$$

Indeed, if not, we must have

$$
\frac{d}{d t} \ln (A / C) \sim \eta / A^{2}
$$

for some $\eta>0$. Hence $\int_{0}^{\infty} \frac{1}{A^{2}}<\infty$. But we also have

$$
\frac{d}{d t} \ln C=\frac{2 C^{2}}{A^{4}} \sim \eta \frac{1}{A^{2}} .
$$

This contradicts the assumption that $\lim _{\infty} C=\infty$. So $\lim _{\infty} A / C=\infty$ as desired.

We have

$$
\frac{d}{d t} \ln \left(A / C^{4}\right)=\frac{2 C(4 A-7 C)}{A^{4}}
$$

Hence $A / C^{4}$ has a positive lower bound, say $\eta^{2}>0$. Now since

$$
\frac{d}{d t} \frac{1}{A^{1 / 2}} \sim-4 \frac{C}{A^{7 / 2}},
$$

we have $\int_{0}^{\infty} \frac{C}{A^{7 / 2}}<\infty$. However

$$
\frac{d}{d t} C=2 \frac{C^{2}}{A^{1 / 2}} \times \frac{C}{A^{7 / 2}}
$$

and $\lim _{\infty} C=\infty$. It follows that

$$
\int_{0}^{\infty} \frac{C}{A^{7 / 2}}>\frac{1}{\eta} \int_{0}^{\infty} \frac{C^{2}}{\sqrt{A}} \frac{C}{A^{7 / 2}}=\infty .
$$

This is a contradiction and we have proved that $\lim _{\infty} C<\infty$.

Now set $\lim _{\infty} C=C_{\infty}$. Then we easily check that $A(t) \sim\left(24 C_{\infty} t\right)^{1 / 3}$, and $C(t)-C_{\infty} \sim-2^{-3} 3^{-1 / 3} C_{\infty}^{5 / 3} t^{-1 / 3}$.

Case 3: $A_{0}>B_{0} \geq C_{0}$.

By Lemma 3.2, the condition $A_{0}>B_{0}$ implies that $A>B$ as long as the solution exits. Assume further that $A_{0} \geq 2 B_{0}$. Again, by Lemma 3.2, we have $A \geq 2 B$ as long as the solution exists.

Lemma 3.4. Assume that $A_{0} \geq 2 B_{0}$. Then $B / C$ is nondecreasing. Furthermore $B, C$ and $B-C$ are non-increasing.

Proof. As $A \geq 2 B$, we have $X>0$ and $A-B-C>0$. The desired conclusions thus follow from (3.4), (3.6) and (3.1). 
Lemma 3.5. Assume $A_{0} \geq 2 B_{0}$. There exists a $T<\infty$, such that $\lim _{t \rightarrow T} C(t)=$ $0, \lim _{t \rightarrow T} B(t)=0$ and $\lim _{t \rightarrow T} A(t)=\infty$.

Proof. As $A \geq 2 B$, we have

$$
\frac{3}{4} A^{2}<X<3 A^{2}
$$

It follows that

$$
-\frac{d}{d t} B=2 \frac{B|Z| X}{A^{2} B^{2} C^{2}}>\frac{3}{2 B}
$$

or

$$
-\frac{d}{d t} B^{2}>3
$$

Hence the solution can only exist up to some finite time $T$, i.e., there exists $T<\infty$, such that either $\lim _{T} C=0$ or $\lim _{T} A=\infty$.

Claim 3.1. If $\lim _{t \rightarrow T} C(t)=0$, then $\lim _{t \rightarrow T} B(t)=0$ and $\lim _{t \rightarrow T} A(t)=\infty$.

To prove the claim, suppose $\lim _{T} C=0, \lim _{T} B=B(T)>0$ and $\lim _{T} A=$ $A(T)<\infty$. Since

$$
\frac{d}{d t} C=2 \frac{C X Y}{A^{2} B^{2} C^{2}}
$$

and

$$
0<\lim _{T} \frac{X|Y|}{A^{2} B^{2}}=\lim _{T} \frac{X}{A^{2}} \frac{(A-B)(A+3 B-2 C)}{B^{2}}=\eta_{1}<\infty
$$

we have

$$
-\frac{d}{d t} C^{2} \sim 4 \eta_{1},
$$

and thus $C^{2}(t) \sim 4 \eta(T-t)$. As $t \rightarrow T,|Z| \sim(A-B)^{2}$ has a positive finite limit. By (3.8), $X$ also has a positive finite limit. Hence

$$
-\frac{d}{d t} B^{2}=\frac{4|Z| X}{A^{2} C^{2}} \sim \frac{\eta_{2}}{T-t} .
$$

This contradicts $\lim _{T} B=B(T)>0$.

Now, we either have $\lim _{T} B=0$ or $\lim _{T} A=\infty$ and, in particular, $\lim _{T} \frac{A}{B}=\infty$. Further, as $t \rightarrow T$, we have

$$
X \sim 3 A^{2}, \quad Y \sim-A^{2}, \text { and } Z \sim-A^{2} .
$$

Assume that $\lim _{T} B(t)=B(T)>0$. Observe that

$$
-\frac{d}{d t} \ln (B-C)=\frac{2 X}{A^{2} B^{2} C^{2}}\left[(A-B-C)^{2}+4 B C\right] \sim \frac{6 A^{2}}{B^{2} C^{2}},
$$

hence

$$
\int_{0}^{T} \frac{A^{2}}{B^{2} C^{2}}<\infty
$$


But we also have

$$
\frac{d}{d t} \ln A=\frac{2 Y Z}{A^{2} B^{2} C^{2}} \sim \frac{2 A^{2}}{B^{2} C^{2}} .
$$

As $\lim _{T} A=\infty$, this is a contradiction. It follows that we must have $\lim _{T} B=0$. A similar argument show that $\lim _{T} A=\infty$. This finishes the proof of the claim 3.1.

To finish the proof of Lemma 3.5, it suffices to rule out the case $\lim _{T} C>0$. Assume $\lim _{T} C=C(T)>0$, then $\lim _{T} B=B(T)>0$ and $\lim _{T} A=\infty$. So, as $t \rightarrow T$, we have $X \sim 3 A^{2}, Y \sim-A^{2}$ and $Z \sim-A^{2}$. Hence

$$
\frac{d}{d t} A=\frac{2 A Y Z}{A^{2} B^{2} C^{2}} \sim \eta A^{3}
$$

with $\eta=2 /(B(T) C(T))^{2}$. It follows that

$$
A(t) \sim \frac{1}{\sqrt{2 \eta(T-t)}} .
$$

We also have

$$
\frac{d}{d t} B^{2} \sim-12 \frac{A^{2}}{C(T)^{2}} \sim-\frac{3 B(T)^{2}}{T-t} .
$$

This contradicts $B(T)>0$ and thus, we must have $\lim _{T} C=0$ as desired.

Lemma 3.6. Assume $A_{0} \geq 2 B_{0}$. As $t \rightarrow T$, there exist positive finite constants $\eta_{1}, \eta_{2}$ and $\eta_{3}$ such that

$$
\left\{\begin{array}{l}
A \sim \eta_{1}(T-t)^{-\frac{1}{14}}, \\
B \sim \eta_{2}(T-t)^{\frac{3}{14}}, \\
C \sim \eta_{3}(T-t)^{\frac{3}{14}}
\end{array}\right.
$$

Proof. As a first step in the proof of this lemma we show the following.

Claim 3.2. As $t \rightarrow T$, we have $\lim _{t \rightarrow T} \frac{B}{C}=\eta \in[1, \infty)$.

We have

$$
\left\{\begin{array}{l}
\frac{d A}{d t} \sim 2 \frac{A^{3}}{(B C)^{2}}, \\
\frac{d B}{d t} \sim-6 \frac{A^{2}}{B C^{2}}, \\
\frac{d C}{d t} \sim-6 \frac{A^{2}}{B^{2} C} .
\end{array}\right.
$$

Moreover, by (3.4), there exists $\eta_{1} \in(0, \infty)$ such that

$$
\frac{d}{d t} \ln (B / C)=\frac{8 X}{A^{2} B C^{2}} \frac{B-C}{B}(A-B-C) \sim \eta_{1} \frac{A}{B C^{2}} .
$$

Here we used that $1-\frac{C}{B}$ is increasing under the flow. We also have $-\frac{d}{d t} B \sim 6 \frac{A^{2}}{B C^{2}}$, so $\int_{0}^{T} \frac{A^{2}}{B C^{2}}<\infty$. As $A \geq A_{0}$ this implies that $\int_{0}^{T} \frac{A}{B C^{2}}<\infty$. Hence $\lim _{T} \frac{B}{C}=\eta<\infty$ as claimed. 
Claim 3.3. As $t \rightarrow T$, we have $\lim _{t \rightarrow T} A^{3} B=\eta_{1}$ and $\lim _{t \rightarrow T} A^{3} C=\eta_{2}$ with $\eta_{1}, \eta_{2} \in(0, \infty)$.

To prove this claim, we compute

$$
\frac{d}{d t}\left(A^{\alpha} B\right)=\frac{2 A^{\alpha} B Z}{A^{2} B^{2} C^{2}}(X+\alpha Y) .
$$

In particular, if $\alpha=3$,

$$
\frac{d}{d t}\left(A^{3} B\right)=\frac{8 A^{3} B Z}{A^{2} B^{2} C^{2}}\left[2 B^{2}-C^{2}-B C+A C-2 A B\right]>0 .
$$

In general,

$X+\alpha Y=(3-\alpha) A^{2}-(B-C)^{2}-2 A B-2 A C+3 \alpha B^{2}-\alpha C^{2}+2 \alpha A C-2 \alpha A B-2 \alpha B C$.

In particular, if $\alpha \neq 3$, then as $t \rightarrow T$,

$$
X+\alpha Y \sim(3-\alpha) A^{2} .
$$

For $\alpha=2$, we see that $\frac{d}{d t}\left(A^{2} B\right)<0$ for $t$ close to $T$ because $Z<0$. Hence $A^{2} B$ is bounded from above. Now, Claim 3.2 and the above formula yields

$$
\frac{d}{d t}\left(A^{3} B\right) \sim 8(2-1 / \eta) \frac{A^{4}}{C^{2}} .
$$

Since $\frac{d}{d t} B \sim-6 \frac{A^{2}}{B C^{2}}$, and $\lim B=0$, we have $\int_{0}^{T} \frac{A^{2}}{B C^{2}}<\infty$. As $A^{2} B$ is bounded from above and $A^{4} / C^{2}=\left(A^{2} B\right) \times A^{2} /\left(B C^{2}\right)$, it follows that $\int_{0}^{T} \frac{A^{4}}{C^{2}}<\infty$. Hence

$$
\lim _{T} A^{3} B=\eta_{1} \in(0, \infty) .
$$

As $\lim B / C=\eta$, this also yields $\lim _{T} A^{3} C=\eta_{1} / \eta$ as claimed.

Now using Claim 3.3 and (3.9), we obtain

$$
\frac{d A}{d t} \sim 2 \eta_{1}^{-2} \eta_{2}^{-2} A^{15}
$$

Hence,

$$
\left\{\begin{array}{l}
A \sim \eta_{3}(T-t)^{-\frac{1}{14}} \\
B \sim \eta_{4}(T-t)^{\frac{3}{14}} \\
C \sim \eta_{5}(T-t)^{\frac{3}{14}}
\end{array}\right.
$$

In order to finish the study of the behavior of $(+\mathrm{XCF})$ on $\mathrm{SU}(2)$, we are left to show that if $A_{0}>B_{0} \geq C_{0}$ then there exists a time $t_{0} \geq 0$ such that $A\left(t_{0}\right) \geq 2 B\left(t_{0}\right)$. We start with the following lemma.

Lemma 3.7. If there exist a time $t_{0}$ such that $X\left(t_{0}\right)>0$, then for all time $t \geq t_{0}$, we have

$$
\frac{X}{A^{2}} \geq \frac{X\left(t_{0}\right)}{A\left(t_{0}\right)}=\eta>0
$$


Moreover, there exists a time $t_{0}^{\prime}$ such that $A\left(t_{0}^{\prime}\right) \geq 2 B\left(t_{0}^{\prime}\right)$.

Proof. We first show that $\frac{X}{A^{2}}>0$ for $t>t_{0}$. Let $t_{1}>t_{0}$ be the first time such that $X\left(t_{1}\right)=0$ (if it exists). Recall that $\frac{B}{A}$ and $\frac{C}{A}$ are decreasing. Moreover, for all $t \in\left[t_{0}, t_{1}\right)$, we have

$$
\frac{d}{d t} \ln (B-C)=-\frac{2 X}{A^{2} B^{2} C^{2}}\left[(A-B-C)^{2}+4 B C\right]<0,
$$

hence $\frac{B-C}{A}$ is decreasing as well on this interval. Since

$$
\frac{X}{A^{2}}=3-\frac{(B-C)^{2}}{A^{2}}-\frac{2 B}{A}-\frac{2 C}{A}
$$

$X / A^{2}$ is increasing on $\left[t_{0}, t_{1}\right)$. This contradicts $X\left(t_{1}\right)=0$, hence we have $\frac{X}{A^{2}}>0$, for all $t>t_{0}$. When $X$ is positive, $B-C$ is decreasing and thus $X / A^{2}$ is increasing. It follows that $\frac{X}{A^{2}}>\eta>0$.

Now, assume that for all time $t>t_{0}, A(t)<2 B(t)$. Since $A$ is increasing and $B$ and $C$ are decreasing, we have $A\left(t_{0}\right) \leq A(t)<2 B(t) \leq 2 B\left(t_{0}\right)<\infty$, for $t>t_{0}$. Since

$$
-\frac{d}{d t} B=\frac{2 B|Z| X}{A^{2} B^{2} C^{2}} \geq \frac{\eta^{\prime}}{B}
$$

for some $\eta^{\prime}>0$, the solution can only exist up to some finite time $T<\infty$. This means that $\lim _{T} C=0$. Since $\lim _{T} A=A(T)$ and $\lim _{T} B=B(T)$ are positive and finite, as $t \rightarrow T$, there is $\eta_{1}>0$ such that

$$
\frac{d}{d t} C^{2} \sim-\eta_{1}
$$

Hence $C \sim \sqrt{\eta_{1}(T-t)}$. This implies

$$
\frac{d}{d t} B^{2} \sim-\frac{\eta_{2}}{T-t}
$$

This contradicts the fact that $B^{2} \geq 0$.

Our final task is to show that it is not possible that $A<2 B$ and $X \leq 0$ for all $t$. To this end, assume that $A<2 B$ and $X \leq 0$ on the interval $[0, T)$ on which the solution exists ( $T$ might be $\infty$ ). Then $A, B, A / B$ and $C$ are all non-decreasing on $[0, T)$. We claim that

$$
\sup _{[0, T)}\{B / C\}=\eta<\infty .
$$

Otherwise, since $B<A$ and $B / A$ is non-increasing, we have

$$
\limsup _{t \rightarrow T} \frac{X}{A^{2}}=\lim _{t \rightarrow T}\left(3-\frac{2 B}{A}-\frac{B^{2}}{A^{2}}+\frac{-C^{2}+2 B C-2 A C}{A^{2}}\right)>0,
$$

which contradicts $X \leq 0$.

Hence we have $A<2 B<2 \eta C$. Observe that $Y, Z$ are negative and

$$
|Y|=A^{2}+C^{2}-2 A C+2 A B+2 B C-3 B^{2}<A^{2}+C^{2}+2 A B+2 B C<11 B^{2},
$$


and

$$
|Z|=A^{2}+B^{2}-2 A B+2 B C+2 A C-3 C^{2}<A^{2}+B^{2}+2 B C+2 A C<\eta_{1} C^{2},
$$

for some $\eta_{1} \in(0, \infty)$. It follows that

$$
\frac{d}{d t} A=\frac{2 A Y Z}{A^{2} B^{2} C^{2}}<\eta_{2} \frac{1}{A}
$$

for some $\eta_{2} \in(0, \infty)$. This implies that $T=\infty$, i.e., the solution exists for all time. We also have

$$
|X|=B^{2}+C^{2}+2 A B+2 A C-2 B C-3 A^{2}<6 A^{2} .
$$

Hence

$$
\frac{d}{d t} B=\frac{2 B Z X}{A^{2} B^{2} C^{2}}<\eta_{3} \frac{1}{B}
$$

for some $\eta_{3} \in(0, \infty)$ and $B^{2} \leq B_{0}^{2}+2 \eta_{3} t$. Now, since

$$
\frac{d}{d t} \ln (A / B)=\frac{8|Z|}{A^{2} B^{2} C^{2}}(A-B)(A+B-C) \geq \frac{8}{B^{2}}\left(1-\frac{B}{A}\right) \geq \frac{\eta_{4}}{B^{2}},
$$

we have

$$
\ln (A / B)-\ln \left(A_{0} / B_{0}\right) \geq \eta_{4} \int_{0}^{t} \frac{1}{B^{2}} \geq \eta_{4} \int_{0}^{t} \frac{d s}{B_{0}^{2}+2 \eta_{3} s} .
$$

The right hand side goes to $\infty$ as $t \rightarrow \infty$, while we have

$$
\lim _{t \rightarrow \infty} \ln (A / B) \leq \ln 2 .
$$

This is the desired contradiction. The conclusion is that, if $A_{0}>B_{0} \geq C_{0}$ then there must exists a time $t_{0}$, such that $A\left(t_{0}\right) \geq 2 B\left(t_{0}\right)$.

THEOREM 3.8. On $\mathrm{SU}(2)$, for the positive cross curvature flow $(+\mathrm{XCF})$ and any choice of initial data $A_{0} \geq B_{0} \geq C_{0}>0$, we have:

- If $A_{0}=B_{0}=C_{0}$, then the solution exists for all time $t$, and we have

$$
A(t)=B(t)=C(t)=\sqrt{A_{0}^{2}+4 t} .
$$

- If $A_{0}=B_{0}>C_{0}$, then the solution exists for all time $t$ and $\lim _{t \rightarrow \infty} C(t)=$ $C_{\infty} \in(0, \infty)$. Moreover, as $t$ tends to $\infty$, we have

$$
A(t)=B(t) \sim\left(24 C_{\infty} t\right)^{1 / 3}, C(t)-C_{\infty} \sim-2^{-3} 3^{-1 / 3} C_{\infty}^{5 / 3} t^{-1 / 3} .
$$

- if $A_{0}>B_{0} \geq C_{0}$, then there exists a finite time $T>0$, such that the solution exists on $[0, T)$ and, as $t \rightarrow T$,

$$
A \sim \eta_{1}(T-t)^{-\frac{1}{14}}, B \sim \eta_{2}(T-t)^{\frac{3}{14}}, \text { and } C \sim \eta_{3}(T-t)^{\frac{3}{14}},
$$

for some finite positive constants $\eta_{1}, \eta_{2}, \eta_{3}$. 
4. The cross curvature flow on $E(1,1)$ (Sol geometry). A model for the geometry $E(1,1)$ is the group $\mathbb{R} \ltimes \mathbb{R}^{2}$ where the action of $\mathbb{R}$ on $\mathbb{R}^{2}$ is given by $\left(\begin{array}{cc}e^{x} & 0 \\ 0 & e^{-x}\end{array}\right)$. This group is sometimes called Sol. In other words, Sol is $\mathbb{R}^{3}$ with the multiplication law

$$
(x, y, z) \cdot\left(x^{\prime}, y^{\prime}, z^{\prime}\right)=\left(x+x^{\prime}, y+e^{x} y^{\prime}, z+e^{-x} z^{\prime}\right) .
$$

If we denote by $X, Y, Z$ the left-invariant vector fields equal to $\partial / \partial x, \partial / \partial y$ and $\partial / \partial z$ at $(0,0,0)$ then $X=\partial / \partial x, Y=e^{x} \partial / \partial y$ and $Z=e^{-x} \partial / \partial z$. Hence, $[Y, Z]=0,[X, Y]=$ $Y,[X, Z]=-Z$. For any left-invariant metric equal to $a(d x)^{2}+b(d y)^{2}+c(d z)^{2}$ at $(0,0,0)$, a Milnor frame is $f_{1}=Y+Z, f_{2}=2 X, f_{3}=Y-Z$. Conversely, given a metric $g_{0}$ and a Milnor frame for that metric, we can define elements $X, Y, Z$ of the Lie algebra by $X=(1 / 2) f_{2}, Y=(1 / 2)\left(f_{1}+f_{3}\right)$ and $Z=(1 / 2)\left(f_{1}-f_{3}\right)$. In the coordinate system induced by the exponential map and the basis $X, Y, Z$, the group law has the form given above. It is useful to observe that, for any $r>0$, the metrics

$$
g=a f^{1} \otimes f^{1}+b f^{2} \otimes f^{2}+c f^{3} \otimes f^{3} \text { and } g=r a f^{1} \otimes f^{1}+b f^{2} \otimes f^{2}+r c f^{3} \otimes f^{3}
$$

yields isometric manifolds, the isometry being induced by the Lie algebra isomorphism $f_{1} \mapsto \sqrt{r} f_{1}, f_{2} \mapsto f_{2}, f_{3} \mapsto \sqrt{r} f_{3}$ and its inverse.

For later purpose, we introduce the manifold

$$
E_{0}(1,1)=E(1,1) / \mathbb{X}
$$

where $\mathbb{X}$ stands for the copy of the integers $\{(k, 0,0): k \in \mathbb{Z}\}$ sitting in $E(1,1)$. Any left-invariant metric on $E(1,1)$ induces a locally homogeneous Riemannian structure on $E_{0}(1,1)$. Moreover, the remark made above concerning isometric structures on $E(1,1)$ is also valid for $E_{0}(1,1)$. We will be particularly interested in those metric $g_{\beta}$, $\beta>0$, of the form

$$
g_{\beta}=\beta f^{2} \otimes f^{2}+f^{1} \otimes f^{1}+f^{3} \otimes f^{3} .
$$

Given a left-invariant metric $g_{0}$, we fix a Milnor frame $\left\{f_{i}\right\}_{1}^{3}$ such that

$$
\left[f_{2}, f_{3}\right]=2 f_{1}, \quad\left[f_{3}, f_{1}\right]=0, \quad\left[f_{1}, f_{2}\right]=-2 f_{3} .
$$

The sectional curvatures are:

$$
\begin{gathered}
K\left(f_{2} \wedge f_{3}\right)=\frac{(A-C)^{2}-4 A^{2}}{A B C}, \\
K\left(f_{3} \wedge f_{1}\right)=\frac{(A+C)^{2}}{A B C}, \\
K\left(f_{1} \wedge f_{2}\right)=\frac{(A-C)^{2}-4 C^{2}}{A B C} .
\end{gathered}
$$

We first state the theorem describing the forward and backward asymptotic behaviors of $(-\mathrm{XCF})$ on $E(1,1)$. Because of the symmetry between $f_{1}$ and $f_{3}$, one can assume that $A \geq C$. 
Theorem 4.1. $\operatorname{Let} g(t)=A(t) f^{1} \otimes f^{1}+B(t) f^{2} \otimes f^{2}+C(t) f^{3} \otimes f^{3}, t \in\left(-T_{b}, T_{f}\right)$, be a maximal solution of the negative cross curvature flow (-XCF) on a complete locally homogeneous manifold of type $E(1,1)$. Assume that $A_{0} \geq C_{0}$ and set

$$
\bar{g}(t)=\frac{B_{0}}{B(t)} g(t) .
$$

- The time $T_{f}$ is finite. If $M$ is compact, as $t \rightarrow T_{f}$, the metric space $(M, \bar{g}(t))$ converges in the Gromov-Hausdorff sense to $\left(E_{0}(1,1), g_{\beta}\right)$ with $g_{\beta}$ as in (4.1) for some $\beta>0$. If $M=E(1,1)$, then the metric space $(E(1,1), \bar{g}(t))$ converges in the Gromov-Hausdorff sense to $\left(E(1,1), g_{\beta}\right)$ for some $\beta>0$.

- If $A_{0}=C_{0}$, then $T_{b}=\infty$ and $A(t)=C(t)=A_{0} B_{0} / B(t), B(t)=\sqrt{B_{0}^{2}-64 t}$. If $M$ is compact, as $t \rightarrow-T_{b}=-\infty$, the metric space $(M, \bar{g}(t)$ ) (resp. $(M, g(t)))$ converges in the Gromov-Hausdorff sense to a circle (resp. to a line).

- If $A_{0}>C_{0}$, then $T_{b}<\infty$ and, as $t \rightarrow-T_{b}$, the metric space $(M, \bar{g}(t))$ converges uniformly to the sub-Riemannian metric space $\left(M, b f_{2} \otimes f_{2}+c f_{3} \otimes\right.$ $\left.f_{3}\right)$ with $b, c \in(0, \infty)$.

Proof. Theorem 2 from [CNSC08] gives that $T_{f}$ is finite and $A, C \sim E_{1} / \sqrt{T_{f}-t}$, $A-C \sim E_{2} \sqrt{T_{f}-t}, B \sim \sqrt{64\left(T_{f}-t\right)}$, as $t$ tends to $T_{f}$. The desired convergence follows. For the behavior when $t$ tends to $-T_{b}$, see Theorem 4.5 below. $\square$

The rest of this section is devoted to the asymptotic behavior of the flow $(* \mathrm{XCF})$ on $E(1,1)$. From the sectional curvature given above, we obtain the equations

$$
\left\{\begin{array}{l}
\frac{d A}{d t}=\frac{2 A Y Z}{(A B C)^{2}} \\
\frac{d B}{d t}=\frac{2 B Z X}{(A B C)^{2}} \\
\frac{d C}{d t}=\frac{2 C X Y}{(A B C)^{2}}
\end{array}\right.
$$

where

$$
\begin{aligned}
& X=(A+C)(3 A-C), \\
& Y=-(A+C)^{2}, \\
& Z=-(A+C)(A-3 C) .
\end{aligned}
$$

First, consider the case when $A_{0}=C_{0}$. Then $A(t)=C(t)$ as long as the solution exists and

$$
\frac{d B}{d t}=\frac{32}{B}
$$

Hence $B=\sqrt{B_{0}^{2}+64 t}$. It follows that

$$
\frac{d \ln A}{d t}=-\frac{32}{B_{0}^{2}+64 t}
$$

which gives

$$
A(t)=C(t)=\frac{A_{0} B_{0}}{\sqrt{B_{0}^{2}+64 t}} .
$$


Second, we assume that $A_{0} \neq C_{0}$. Because of the symmetry between $f_{1}$ and $f_{3}$, we may assume without loss of generality that $A_{0}>C_{0}$. It immediately follows that $C$ is decreasing.

Lemma 4.2. Assume that $A_{0}>C_{0}$. Then $C$ is decreasing, $A-C, A / C$ and $A-3 C$ are increasing as long as the solution exists. In particular, we have $A>C$.

Proof. Observe that

$$
\begin{aligned}
& \frac{d(A-C)}{d t}=2 \frac{(A+C)^{4}}{(A B C)^{2}}(A-C) \\
& \frac{d \ln (A / C)}{d t}=8 \frac{(A+C)^{3}}{(A B C)^{2}}(A-C) \\
& \frac{d(A-3 C)}{d t}=2 \frac{(A+C)^{3}}{(A B C)^{2}}\left(A^{2}+6 A C-3 C^{2}\right) .
\end{aligned}
$$

The stated results follow. $\square$

Lemma 4.3. Assume that $A_{0}>3 C_{0}$. Then $A$ is increasing, $B$ is decreasing. Moreover, there exists a finite time $T$ such that

$$
\lim _{t \rightarrow T} A(t)=\infty, \lim _{t \rightarrow T} B(t)=0, \lim _{t \rightarrow T} C(t)=0 .
$$

Proof. The previous lemma shows that $A / C>A_{0} / C_{0}>3$ as long as the solution exists. The monotonicity of $A$ and $B$ immediately follows. Further, we have

$$
-B \frac{d}{d t} B=2 \frac{(3 A-C)(A-3 C)(A+C)^{2}}{A^{2} C^{2}}>4\left(1-\frac{3 C}{A}\right)\left(1+\frac{A}{C}\right)^{2}>\eta .
$$

This implies that the solution can only exist up to some finite time $T$. As

$$
\frac{d}{d t} \ln (B / C)=\frac{8(A+C)^{2}(3 A-C)}{A^{2} B^{2} C}>0,
$$

$B / C$ is increasing. Hence if $\lim _{t \rightarrow T} B(t)=0$, then $\lim _{t \rightarrow T} C(t)=0$ as well.

Assume that we have $\lim _{T} A=A(T)<\infty$ and $\lim _{T} B=B(T)>0$. Then we must have $\lim _{T} C=0$ (otherwise, the solution could be extended past $T$ ). As

$$
\frac{d}{d t} C^{2}=\frac{4 X Y}{(A B)^{2}}
$$

it then follows that $C(t)^{2} \sim \eta_{1}(T-t)$, for some positive finite $\eta_{1}$. This implies

$$
\frac{d}{d t} A=\frac{2 A Y Z}{(A B C)^{2}} \sim \frac{\eta_{2}}{T-t}
$$

and thus $A \rightarrow \infty$. This is a contradiction.

Assume next that $\lim _{T} A=A(T)<\infty$ and $\lim _{T} B=0$. Then $\lim _{T} C=0$ and

$$
\begin{gathered}
B^{2} \frac{d}{d t} C^{2}=-\frac{4(A+C)^{3}(3 A-C)}{A^{2}} \sim-12 A^{2}, \\
C^{2} \frac{d}{d t} B^{2}=\frac{4(3 A-C)(3 C-A)(A+C)^{2}}{A^{2}} \sim-12 A^{2} .
\end{gathered}
$$


Hence

$$
\frac{d}{d t}\left(B^{2} C^{2}\right) \sim-24 A^{2}
$$

and $B^{2} C^{2} \sim \eta_{3}(T-t)$ with $\eta_{3} \in(0, \infty)$. Plugging this into the formula for $\frac{d}{d t} A$ shows that $A \rightarrow \infty$, which is a contradiction.

This shows that, as stated in Lemma $4.3 \lim _{T} A=\infty$. To see that $\lim _{T} B=0$, we compute

$$
\frac{d}{d t}\left(A^{\alpha} B\right)=\frac{2 A^{\alpha} B Z}{A^{2} B^{2} C^{2}}(X+\alpha Y) .
$$

If $0<\alpha<3$, then as $t \rightarrow T$,

$$
X+\alpha Y=(A+C)[(3-\alpha) A-(1+\alpha) C]>0 .
$$

As $Z$ is negative, this implies that $\frac{d}{d t} A^{\alpha} B<0$. Thus $A^{\alpha} B$ is decreasing. Since $A \rightarrow \infty$, we also have $B \rightarrow 0$ and thus $C \rightarrow 0$. $\square$

Lemma 4.4. Assume that $A_{0}>3 C_{0}$ and let $T$ be as in Lemma 4.3. Then there are positive finite constants $\eta_{i}, i=1,2,3$ such that, as $t$ tends to $T$,

$$
A \sim \eta_{1}(T-t)^{-\frac{1}{14}}, B \sim \eta_{2}(T-t)^{\frac{3}{14}}, C \sim \eta_{3}(T-t)^{\frac{3}{14}} .
$$

Proof. Taking $\alpha=3$ in (4.3) yields

$$
\frac{d}{d t}\left(A^{3} B\right)=\frac{2 A Z}{B C^{2}}(3 Y+X)=-\frac{8 A Z}{B C}(A+C)>0 .
$$

Similarly,

$$
\frac{d}{d t}\left(A^{3} C\right)=\frac{2 A Y}{B^{2} C}(3 Z+X)=-\frac{16 A}{B^{2}}(A+C)^{3}<0 .
$$

Now, the technique used in the proof of Claim 3.3 shows that

$$
\lim _{t \rightarrow T} A^{3} B<\infty \text { and } \lim _{t \rightarrow T} A^{3} C>0 .
$$

As $t$ tends to $T$, this yields

$$
\frac{d A}{d t} \sim \eta_{1} A^{15}, \frac{d B}{d t} \sim-\eta_{2} B^{-\frac{11}{3}}, \frac{d C}{d t} \sim-\eta_{3} C^{-\frac{11}{3}},
$$

for positive finite $\eta_{i}, i=1,2,3$. The asymptotic stated in Lemma 4.4 follows.

We are left with the task to rule out the possibility that $C<A \leq 3 C$ as long as the solution exists. Assume that $C<A \leq 3 C$. Then $A$ and $C$ are decreasing and $B$ is increasing. Since we have

$$
B \frac{d}{d t} B=2 \frac{(3 A-C)(3 C-A)(A+C)^{2}}{(A C)^{2}}<\eta,
$$

we have

$$
B \leq \sqrt{B_{0}^{2}+2 \eta t}
$$


It follows that

$$
\frac{d}{d t} \ln (A / C)=8 \frac{(A+C)^{3}}{A^{2} C} \frac{1}{B^{2}}\left(\frac{A}{C}-1\right)>\frac{\eta}{B_{0}^{2}+2 \eta t} .
$$

As $\ln (A / C) \leq \ln 3$, the solution can only exist up to some finite time $T$, i.e., there exist $T<\infty$, such that $\lim _{t \rightarrow T} A(t)=\lim _{t \rightarrow T} C(t)=0$. Hence $\lim _{t \rightarrow T}(A-C)(t)=0$. This contradicts the fact that $A-C$ is increasing. It follows that there must exist a finite time $t_{0}$, such that $A\left(t_{0}\right)>3 C\left(t_{0}\right)$. We have proved the following theorem.

THEOREM 4.5. On $E(1,1)$, for any given initial data $A_{0}, B_{0}, C_{0}>0$ with $A_{0} \geq$ $C_{0}$, the positive cross curvature flow behaves as follows.

- If $A_{0}=C_{0}$, then the solution of $(+\mathrm{XCF})$ exists on $[0, \infty)$ and is given by

$$
A(t)=C(t)=\frac{A_{0} B_{0}}{\sqrt{B_{0}^{2}+64 t}}, \quad B(t)=\sqrt{B_{0}^{2}+64 t} .
$$

- If $A_{0}>C_{0}$, then there exists a positive finite time $T_{b}$ such that the solution of $(+\mathrm{XCF})$ exists on $\left[0, T_{b}\right)$. Moreover, as $t \rightarrow T_{b}$,

$$
A \sim \eta_{1}(T-t)^{-\frac{1}{14}}, B \sim \eta_{2}(T-t)^{\frac{3}{14}}, \text { and } C \sim \eta_{3}(T-t)^{\frac{3}{14}}
$$

with $\eta_{1}, \eta_{2}, \eta_{3} \in(0, \infty)$.

5. The cross curvature flow on $E(2)$. Recall that a realization of $\widetilde{E(2)}$ is $\mathbb{R}^{2} \rtimes \mathbb{R}$ where the action of $\mathbb{R}$ on $\mathbb{R}^{2}$ is by rotation. Namely, $\widetilde{E(2)}$ is $\mathbb{R}^{3}$ equipped with the product

$$
(x, y, z) \cdot\left(x^{\prime}, y^{\prime}, z^{\prime}\right)=\left(x+x^{\prime} \cos z+y^{\prime} \sin z, y+x^{\prime} \cos z-y^{\prime} \sin z, z+z^{\prime}\right) .
$$

Note that the left-invariant metrics on $\widetilde{E(2)}$ equal to $a\left[(d x)^{2}+(d y)^{2}\right]+c(d z)^{2}$ at the identity element are actually flat metrics on $\mathbb{R}^{3}$.

Given a left-invariant metric $g_{0}$, we fix a Milnor frame $\left\{f_{i}\right\}_{1}^{3}$ such that

$$
\left[f_{2}, f_{3}\right]=2 f_{1}, \quad\left[f_{3}, f_{1}\right]=2 f_{2}, \quad\left[f_{1}, f_{2}\right]=0 .
$$

These relations imply that, in the global coordinate introduce above, $f_{1}, f_{2}$ are in the span of $\partial / \partial x, \partial / \partial y$ whereas $f_{3}$ contains a $\partial / \partial z$ component.

The sectional curvatures are:

$$
\begin{gathered}
K\left(f_{2} \wedge f_{3}\right)=\frac{1}{A B C}(B-A)(B+3 A), \\
K\left(f_{3} \wedge f_{1}\right)=\frac{1}{A B C}(A-B)(A+3 B), \\
K\left(f_{1} \wedge f_{2}\right)=\frac{1}{A B C}(A-B)^{2} .
\end{gathered}
$$

As in previous sections, we first state a theorem that describes both the forward and backward behavior of the negative cross curvature flow on $E(2)$. The forward 
behavior was studied in [CNSC08, sect. 6]. The backward behavior is studied below in the form of the forward behavior of the positive cross curvature flow. Because of the symmetry between $f_{1}$ and $f_{2}$ we can assume that $A_{0} \geq B_{0}$.

Theorem 5.1. $\operatorname{Let} g(t)=A(t) f^{1} \otimes f^{1}+B(t) f^{2} \otimes f^{2}+C(t) f^{3} \otimes f^{3}, t \in\left(-T_{b}, T_{f}\right)$, be a maximal solution of the negative cross curvature flow on a complete locally homogeneous manifold of type E(2). Set

$$
\bar{g}(t)=\frac{C_{0}}{C(t)} g(t) .
$$

- Assume that $A_{0}=B_{0}$. Then $T_{b}=T_{f}=\infty, A(t)=B(t)=A_{0}$ and $C(t)=C_{0}$ for all $t$. In fact, as a Riemannian manifold, $(\widetilde{E(2)}, g)$ is $\mathbb{R}^{3}$ equipped with a flat metric.

- Assume that $A_{0}>B_{0}$. Then $T_{f}=\infty$ and $T_{b}<\infty$.

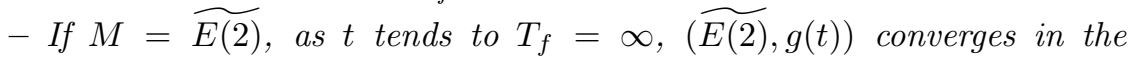
Gromov-Hausdorff sense to $\mathbb{R}^{3}$ (the sectional curvatures go to zero). If $M$ is compact, $(M, g(t))$ does not converge to a metric space as $t$ tends to infinity but $(M, \bar{g}(t))$ converge in the Gromov-Hausdorff sense to a circle.

- In all cases, as t tends to $-T_{b},(M, \bar{g}(t))$ converges uniformly to the subRiemannian metric space $\left(E(2), b f_{2} \otimes f_{2}+c f_{3} \otimes f_{3}\right)$ for some $b, c \in(0, \infty)$.

Proof. The first case is trivial. When $A_{0}>B_{0}$, the results in the forward direction follow from Theorem 5 of [CNSC08] which gives $T_{f}=\infty$ and $A \sim E_{1}+E_{2} t^{-1 / 6}$, $B \sim E_{1}-E_{2} t^{-1 / 6}$ and $C \sim\left(8 \sqrt{6} E_{2} / E_{1}\right) t^{1 / 3}$, as $t$ tends to infinity.

The result in the backward direction follow from Theorem 5.4 below. $\square$

REMARK 5.1. For $t>0$, in the orthonormal frame $\left(e_{i}^{t}\right) 1^{3}$ on $\left.\widetilde{(E(2)}, g(t)\right)$, with $e_{i}^{t}$ positively collinear to $f_{i}$, we have $\left[e_{2}^{t}, e_{3}^{t}\right] \sim \delta t^{-1 / 6} e_{1}^{t},\left[e_{3}^{t}, e_{1}^{t}\right] \sim \delta t^{-1 / 6} e_{2}^{t},\left[e_{1}^{t}, e_{2}^{t}\right]=0$ for some $\delta>0$. In other words, the group structure viewed in this frame tends to the abelian structure of $\mathbb{R}^{3}$. This is similar to what happens on the Heisenberg group. Compare Remark 2.3.

The rest of this section is devoted to the study of the positive cross curvature $(+\mathrm{XCF})$ on $E(2)$. Hence $g(t)=A(t) f^{1} \otimes f^{1}+B(t) f^{2} \otimes f^{2}+C(t) f^{3} \otimes f^{3}$ is a solution of $(+\mathrm{XCF})$ and thus $A, B, C$ satisfy the following equations

$$
\left\{\begin{array}{l}
\frac{d A}{d t}=\frac{2 A Y Z}{(A B C)^{2}} \\
\frac{d B}{d t}=\frac{2 B Z X}{(A B C)^{2}} \\
\frac{d C}{d t}=\frac{2 C X Y}{(A B C)^{2}}
\end{array}\right.
$$

where

$$
\begin{aligned}
& X=(A-B)(3 A+B), \\
& Y=(B-A)(3 B+A), \\
& Z=-(A-B)^{2} .
\end{aligned}
$$


If $A_{0}=B_{0}$, then $A=A_{0}, B=B_{0}$ and $C=C_{0}$. The geometry stays flat all time. Without loss of generality, we assume that $A_{0}>B_{0}$. Then $A$ is increasing, while $B$ and $C$ are decreasing.

Lemma 5.2. Assume $A_{0}>B_{0}$. Then there is a finite time $T$ such that the solution exists on $[0, T)$ and

$$
\lim _{t \rightarrow T} A(t)=\infty, \lim _{t \rightarrow T} B(t)=0 \text { and } \lim _{t \rightarrow T} C(t)=0 .
$$

Proof. Notice that

$$
X>\eta A^{2},|Y|>\eta A^{2} \text { and }|Z|>\eta A^{2}
$$

for some constant $\eta \in(0, \infty)$. Hence

$$
-\frac{d}{d t} C>\frac{2 \eta^{2} A^{4}}{A^{2} B^{2} C}>\eta_{1}>0 .
$$

It follows that the solution exists up to some finite time $T \in(0, \infty)$. Next

$$
\frac{d}{d t} \ln (A B)=\frac{2 Z}{A^{2} B^{2} C^{2}}(X+Y)=\frac{-4(A-B)^{4}}{A^{2} B^{2} C^{2}}<0,
$$

hence $A B$ is decreasing. Similarly,

$$
\frac{d}{d t} \ln (A C)=\frac{2 Y}{A^{2} B^{2} C^{2}}(X+Z)<0,
$$

so $A C$ is also decreasing.

We first show that $\lim _{T} C=0$. Assume not. Then $\lim _{T} C=C(T) \in(0, \infty)$. Since $A C$ is decreasing, we get $\lim _{T} A=A(T)<\infty$ while $\lim _{T} B=0$. As $t \rightarrow T$, we have

$$
X \sim 3 A^{2}, Y \sim-A^{2}, Z \sim-A^{2},
$$

hence

$$
\frac{d}{d t} B^{2}=\frac{4 Z X}{A^{2} C^{2}} \sim-\eta
$$

for some positive fine $\eta$. It follows that $B \sim \sqrt{\eta(T-t)}$ and

$$
\frac{d}{d t} A=\frac{2 A Y Z}{(A B C)^{2}} \sim \frac{\eta}{T-t}
$$

for a different $\eta \in(0, \infty)$. This shows that $A(t) \sim-\eta \ln (T-t) \rightarrow \infty$ as $t \rightarrow T$, which is a contradiction.

Next we show that $\lim _{T} A=\infty$. Assume that we have $\lim _{T} A=A(T)<\infty$. If further more we have $\lim _{T} B=B(T)>0$ then, since

$$
\frac{d}{d t} C^{2}=\frac{4 X Y}{(A B)^{2}} \sim-\eta,
$$

and thus $C(t)^{2} \sim \eta(T-t)$, just as above, we can show that $A \rightarrow \infty$, this is a contradiction. 
If we have $\lim _{T} B=0$, then

$$
B^{2} \frac{d}{d t} C^{2}=\frac{4 X Y}{A^{2}} \sim-12 A(T)^{2},
$$

and

$$
C^{2} \frac{d}{d t} B^{2}=\frac{4 X Z}{A^{2}} \sim-12 A(T)^{2} .
$$

Hence $\frac{d}{d t}\left(B^{2} C^{2}\right) \sim-24 A(T)^{2}$ and thus $B^{2} C^{2} \sim 24 A(T)^{2}(T-t)$. This again leads to $A \rightarrow \infty$, which is a contradiction. This proves that $\lim _{T} A=\infty$.

Since $A B$ is decreasing, so we have proved that $\lim _{T} A=\infty$ and $\lim _{T} B=$ $\lim _{T} C=0 . \square$

Lemma 5.3. Assume that $A_{0}>B_{0}$. Then there are constants $\eta_{1}, \eta_{2}, \eta_{3} \in(0, \infty)$ such that

$$
A \sim \eta_{1}(T-t)^{-\frac{1}{14}}, \quad B \sim \eta_{2}(T-t)^{\frac{3}{14}}, C \sim \eta_{3}(T-t)^{\frac{3}{14}}
$$

Proof. As

$$
\frac{d}{d t}\left(A^{3} B\right)=-\frac{16 A Z}{C^{2}}(A-B)>0 \text { and } \frac{d}{d t}\left(A^{3} C\right)=\frac{8 A Y}{B C}(A-B)<0,
$$

it follows that $A^{3} B$ is increasing and $A^{3} C$ decreasing. We also have

$$
\frac{d}{d t}\left(A^{2} B\right)=\frac{2 A^{2} B Z}{A^{2} B^{2} C^{2}}(X+2 Y) .
$$

As $t \rightarrow T, X+2 Y=(A-B)(A-5 B)>0$. This implies that $A^{2} B$ is bounded from above. Using the same technique as in the proof of Claim 3.3, we obtain that there exists $\eta \in(0, \infty)$ such that $\lim _{T} A^{3} B=\eta$. Finally, observe that

$$
\frac{d}{d t} \ln \left(A^{3} C\right) \sim-8 \frac{A}{B C^{2}} \text { and } \frac{d}{d t} B \sim-6 \frac{A^{2}}{B C^{2}} .
$$

Since $B>0$ and $\lim _{T} A=\infty$, it follows that $\int^{T} A /\left(B C^{2}\right) \leq \int^{T} A^{2} /\left(B C^{2}\right)<\infty$. Hence $\lim _{T} A^{3} C=\eta^{\prime} \in(0, \infty)$. These asymptotic behaviors of $A^{3} B$ and $A^{3} C$ imply that

$$
\frac{d A}{d t} \sim \eta A^{15}, \frac{d B}{d t} \sim-\eta B^{-\frac{11}{3}}, \frac{d C}{d t} \sim-\eta C^{-\frac{11}{3}} .
$$

The desired asymptotic results follow.

THEOREM 5.4. Let $g(t)$ be the solution of $(+\mathrm{XCF})$ on $E(2)$ with given initial data $A_{0}, B_{0}, C_{0}>0$ in a Milnor frame $f_{1}, f_{2}, f_{3}$ as above.

- Assume that $A_{0}=B_{0}$. Then $g(t)=g_{0}=A_{0} f^{1} \otimes f^{1}+A_{0} f^{2} \otimes f^{2}+C_{0} f^{3} \otimes f^{3}$.

- If $A_{0}>B_{0}$, then there exists a finite time $T>0$, such that $g(t)$ exists on $[0, T)$ and, as $t \rightarrow T$,

$$
A \sim \eta_{1}(T-t)^{-\frac{1}{14}}, B \sim \eta_{2}(T-t)^{\frac{3}{14}}, \text { and } C \sim \eta_{3}(T-t)^{\frac{3}{14}}
$$

for some constants $\eta_{1}, \eta_{2}, \eta_{3} \in(0, \infty)$. 
6. The cross curvature flow on $\operatorname{SL}(2, \mathbb{R})$. Given a left-invariant metric $g_{0}$ on $\operatorname{SL}(2, \mathbb{R})$, we fix a Milnor frame $\left\{f_{i}\right\}_{1}^{3}$ such that

$$
\left[f_{2}, f_{3}\right]=-2 f_{1}, \quad\left[f_{3}, f_{1}\right]=2 f_{2}, \quad\left[f_{1}, f_{2}\right]=2 f_{3}
$$

and

$$
g_{0}=A_{0} f^{1} \otimes f^{1}+B_{0} f^{2} \otimes f^{2}+C_{0} f^{3} \otimes f^{3} .
$$

The sectional curvatures are

$$
\begin{aligned}
& K\left(f_{2} \wedge f_{3}\right)=\frac{1}{A B C}\left(-3 A^{2}+B^{2}+C^{2}-2 B C-2 A C-2 A B\right), \\
& K\left(f_{3} \wedge f_{1}\right)=\frac{1}{A B C}\left(-3 B^{2}+A^{2}+C^{2}+2 B C+2 A C-2 A B\right), \\
& K\left(f_{1} \wedge f_{2}\right)=\frac{1}{A B C}\left(-3 C^{2}+A^{2}+B^{2}+2 B C-2 A C+2 A B\right) .
\end{aligned}
$$

Recall that the Lie algebra $\operatorname{sl}(2, \mathbb{R})$ of $\operatorname{SL}(2, \mathbb{R})$ can be realized as the space of two by two real matrices with trace 0 . A basis of this space is

$$
W=\left(\begin{array}{cc}
0 & -1 \\
1 & 0
\end{array}\right), \quad H=\left(\begin{array}{cc}
1 & 0 \\
0 & -1
\end{array}\right), \quad V=\left(\begin{array}{ll}
0 & 1 \\
1 & 0
\end{array}\right) .
$$

These satisfy

$$
[H, V]=-2 W,[W, H]=2 V,[V, W]=2 H .
$$

This means that $(W, V, H)$ can be taken as a concrete representation of the above Milnor basis $\left(f_{1}, f_{2}, f_{3}\right)$. In particular, $f_{1}$ corresponds to rotation in $\operatorname{SL}(2, \mathbb{R})$. Note further that exchanging $f_{2}, f_{3}$ and replacing $f_{1}$ by $-f_{1}$ produce another Milnor basis. This explains the $B, C$ symmetry of the formulas above.

As for the other cases, the forward behavior of (XCF) was studied in [CNSC08, sect. 5]. However, in the $\operatorname{SL}(2, \mathbb{R})$ case, the description of this forward asymptotic behavior in terms of convergence of metric spaces becomes quite intricate and we will only make some simple remarks. The reader can consult [Gli08] for a careful analysis using groupoid techniques.

The backward behavior is studied below in the form of the forward behavior of the positive cross curvature flow. Because of the symmetry between $f_{2}$ and $f_{3}$ noted above, we can assume that $B_{0} \geq C_{0}$.

Theorem 6.1. Let $g(t)=A(t) f^{1} \otimes f^{1}+B(t) f^{2} \otimes f^{2}+C(t) f^{3} \otimes f^{3}, t \in\left(-T_{b}, T_{f}\right)$, be a maximal solution of the negative cross curvature flow on a complete locally homogeneous manifold of type $\mathrm{SL}(2, \mathbb{R})$. Then $T_{b}<\infty$ whereas $T_{f}=\infty$ if $B_{0}=C_{0}$ and $T_{f}<\infty$ otherwise. Assume that $B_{0} \geq C_{0}$ and set

$$
Q=\left\{(a, b, c) \in \mathbb{R}^{3}: a>0, b \geq c>0\right\}
$$

and

$$
\bar{g}(t)=\frac{C_{0}}{C(t)} g(t) .
$$

There is a partition of $Q$ into subsets $S_{0}, Q_{1}, Q_{2}$ with $Q_{1}, Q_{2}$ connected and, as $t$ tends to $-T_{b}$ : 
1. If $\left(A_{0}, B_{0}, C_{0}\right) \in Q_{1},(M, \bar{g}(t))$ converges uniformly to the sub-Riemannian metric space $\left(M, b f_{2} \otimes f_{2}+c f_{3} \otimes f_{3}\right)$ for some $b, c \in(0, \infty)$.

2. If $\left(A_{0}, B_{0}, C_{0}\right) \in Q_{2},(M, \bar{g}(t))$ converges uniformly to the sub-Riemannian metric space $\left(M, a f_{1} \otimes f_{1}+c f_{3} \otimes f_{3}\right)$ for some $a, c \in(0, \infty)$.

3. If $(A(t), B(t), C(t)) \in S_{0}$ for all $t \in\left(-T_{b}, 0\right]$ then $A(t)$ tends to 0 whereas $B(t)$ and $C(t)$ converge towards the same finite constant. If $M$ is compact, $(M, \bar{g}(t))$ converges in the Gromov-Hausdorff sense to a compact surface of constant negative curvature.

REMARK 6.1. In the forward direction, if $B_{0}=C_{0}$ and $M$ is compact then $(M, \bar{g}(t))$ converges in the Gromov-Hausdorff sense to a compact surface of constant negative sectional curvature. See [CNSC08] and [Gli08].

Remark 6.2. The cases (1)-(2) of Theorem 6.1 are somewhat symmetric. Case (1) occurs when $A_{0}$ is large compared to $B_{0}-C_{0}$. Case (2) occurs when $A_{0}$ is small compared to $B_{0}-C_{0}$. Case (3) is of a completely different nature and it is not even entirely clear, a priori, that it occurs at all. In a forthcoming work [CGSC09], we will show that $Q_{1} \cup Q_{2}$ is a dense open set and that $S_{0}$ is an hypersurface separating $Q_{1}$ from $Q_{2}$. This however requires different techniques that those used in this paper.

The rest of this section is devoted to the proof of Theorem 6.1. A much more precise statement is given in Theorem 6.12. As in earlier sections, we focus on the forward behavior of solutions of $(+\mathrm{XCF})$. Using the sectional curvatures given above, writing

$$
g=A f^{1} \otimes f^{1}+B f^{2} \otimes f^{2}+C f^{3} \otimes f^{3}
$$

for the solution of the flow $(+\mathrm{XCF})$ with initial data $g_{0}, A, B, C$ satisfy the equations

$$
\left\{\begin{aligned}
\frac{d A}{d t} & =\frac{2 A F_{2} F_{3}}{(A B C)^{2}}, \\
\frac{d B}{d t} & =\frac{2 B F_{3} F_{1}}{(A B C)^{2}}, \\
\frac{d C}{d t} & =\frac{2 C F_{1} F_{2}}{(A B C)^{2}},
\end{aligned}\right.
$$

where

$$
\begin{aligned}
& F_{1}=-3 A^{2}+B^{2}+C^{2}-2 B C-2 A C-2 A B, \\
& F_{2}=-3 B^{2}+A^{2}+C^{2}+2 B C+2 A C-2 A B, \\
& F_{3}=-3 C^{2}+A^{2}+B^{2}+2 B C-2 A C+2 A B .
\end{aligned}
$$

Without loss of generality we may assume that $B_{0} \geq C_{0}$. Then $B \geq C$ as long as a solution exists and

$$
F_{3}=(B-C)(2 A+B+3 C)+A^{2} \geq A^{2}>0 .
$$

Observe also that $F_{1}+F_{2}<0$ so at least one of the quantities $F_{1}, F_{2}$ is negative.

Let $a=A / B$ and $c=C / B$. 
Lemma 6.2. Suppose that $a_{0}=A_{0} / B_{0}$ and $c_{0}=C_{0} / B_{0}$ satisfy

$$
a>1-c+2 \sqrt{1-c}
$$

Then a and c satisfy (6.2) as long as a solution exists. Moreover, in this case, (6.2) is equivalent to $F_{2}>0$ and thus implies that $F_{1}<0$.

Proof. As in [CNSC08, Sect. 5, Lemma 2], we have

$$
F_{2}=(A-(B-C+2 \sqrt{(B-C) B}))(A-(B-C-2 \sqrt{(B-C) B})) .
$$

Since $B-C-2 \sqrt{(B-C) B} \leq 0$, it follows that (6.2) is equivalent to $F_{2}>0$. Observe that

$$
\left.\frac{d A}{d t}\right|_{F_{2}=0}=0,\left.\quad \frac{d B}{d t}\right|_{F_{2}=0}<0 \quad \text { and }\left.\frac{d C}{d t}\right|_{F_{2}=0}=0 .
$$

It follows that

$$
\left.\frac{d F_{2}}{d t}\right|_{F_{2}=0}>0
$$

To prove that $F_{2}(t)>0$ we argue by contradiction. Suppose $t_{0}$ is the first time such that $F_{2}\left(t_{0}\right)=0$. Since $F_{2}(0)>0$, we know that $\frac{d}{d t} F_{2}\left(t_{0}\right) \leq 0$, which contradicts $(6.3)$. Therefore $F_{2}(t)>0$, which is equivalent to (6.2). This completes the proof of the lemma. $\mathrm{c}$

The next lemma is very similar to the previous one and we omit the proof.

Lemma 6.3. Suppose that $a_{0}=A_{0} / B_{0}$ and $c_{0}=C_{0} / B_{0}$ satisfy

$$
a<\frac{1}{3}\left(2 \sqrt{1-c+c^{2}}-1-c\right) .
$$

Then $a$ and $c$ satisfy and (6.4) as long as a solution exists. Moreover, (6.4) is equivalent to $F_{1}>0$ and thus implies $F_{2}<0$.

Observe that, if at any time $t_{0}$, the solution satisfies either (6.2) or (6.4) then that inequality will be satisfied at all later time. We will consider three cases: The case where (6.2) is satisfied (at time $t=0$ or, in fact at a later time), the case where (6.4) is satisfied (at time $t=0$ or, in fact at a later time), and the remaining case where neither (6.2) nor (6.4) is satisfied as long as the solution exists.

Case 1: Inequality (6.2) is satisfied. Recall that this is equivalent to say that $F_{2}>0$. Moreover, we must have $F_{1}<0$.

Lemma 6.4. Assume that $B \geq C$ and (6.2) holds. Then $A$ is increasing, $B$ and $C$ are decreasing and $A+C \geq B$. Furthermore $a, c$ and $F_{2} / B^{2}$ are non-decreasing and $\left|F_{1}\right|>3 A^{2}$.

Proof. The monotonicity of $A, B$ and $C$ follows from the fact that $F_{2}>0$ which also easily imply $A+C \geq B$. Further

$$
\frac{d \ln (A / B)}{d t}=\frac{8(A+B)}{(A B C)^{2}} F_{3}(A+C-B),
$$

and

$$
\frac{d \ln (C / B)}{d t}=\frac{8(B-C)}{(A B C)^{2}}(A+B+C)\left|F_{1}\right|,
$$


are non-negative. As

$$
\frac{F_{2}}{B^{2}}=\left(\frac{A+C}{B}-1\right)^{2}+4 \frac{C}{B}-4
$$

and

$$
\left|F_{1}\right|=3 A^{2}+2(A+C) B+2 A C-B^{2}-C^{2} .
$$

The lemma follows.

Lemma 6.5. Assume that $B \geq C$ and (6.2) is satisfied. Then there exists a finite time $T$ such that

$$
\lim _{t \rightarrow T} A(t)=\infty, \lim _{t \rightarrow T} B(t)=\lim _{t \rightarrow T} C(t)=0 .
$$

Moreover, there exists $\eta_{0} \in(0, \infty)$ such that $\lim _{T} B / C=\eta_{0}$.

Proof. By Lemma 6.4, there exists $\eta \in(0, \infty)$ such that

$$
\frac{d}{d t} C^{2}=-4 \frac{\left|F_{1}\right|}{A^{2}} \times \frac{F_{2}}{B^{2}}<-12 \eta .
$$

Hence there exists a finite time $T$ such that the flow exists only up to $T$ and either $\lim _{T} C=0$ or $\lim _{T} A=\infty$.

Observe that $C / B \leq 1$ is non-decreasing. Hence $\lim _{T} B / C \in(0, \infty)$. We first show that $\lim _{T} C=0$, which implies that $\lim _{T} B=0$. Otherwise we have $\lim _{T} A=\infty$ and $\lim _{T} C=C(T)>0$. Note that $\lim _{T} B=B(T) \geq C(T)>0$. Since

$$
\frac{d}{d t} A=\frac{2}{A} \times \frac{F_{2}}{B^{2}} \times \frac{F_{3}}{C^{2}},
$$

and $F_{2} \sim F_{3} \sim A^{2}$ as $t \rightarrow T$, there exists a constant $\eta>0$, such that

$$
\frac{d}{d t}\left(A^{-2}\right) \sim-\frac{1}{\eta}
$$

Hence

$$
A^{2} \sim \frac{\eta}{T-t}
$$

As $\left|F_{1}\right| \sim 3 A^{2}$,

$$
\frac{d}{d t} C^{2}=-4 \frac{\left|F_{1}\right|}{A^{2}} \frac{F_{2}}{B^{2}} \sim-\frac{12 \eta}{B(T)^{2}} \times \frac{1}{T-t} .
$$

This contradicts the fact that $C$ is decreasing with $C(T)>0$.

Next, we prove that $\lim _{T} A=\infty$. Observe that, for any $\alpha>0$, we have

$$
\frac{d}{d t}\left(A^{\alpha} B\right)=\frac{2 A^{\alpha} B F_{3}}{(A B C)^{2}}\left(\alpha F_{2}+F_{1}\right)
$$

and

(6.9) $\alpha F_{2}+F_{1}=(\alpha-3) A^{2}+(1-3 \alpha) B^{2}+(1+\alpha) C^{2}+2(\alpha-1)(B+A) C-2(1+\alpha) A B$. 
If $\alpha>3$, then $\lim _{T}\left(\alpha F_{2}+F_{1}\right)>0$ and thus $A^{\alpha} B$ increasing. As $\lim _{T} B=0$, it follows that $\lim _{T} A=\infty$, as desired.

Lemma 6.6. Assume that $B \geq C$ and (6.2) holds. There are constants $\eta_{1}, \eta_{2}, \eta_{3} \in$ $(0, \infty)$ such that, as $t$ tends to $T$,

$$
A(t) \sim \eta_{1}(T-t)^{-1 / 14}, \quad B(t) \sim \eta_{2}(T-t)^{3 / 14} \text { and } C(t) \sim \eta_{3}(T-t)^{3 / 14} .
$$

Proof. For $\alpha=3,\left(\alpha F_{2}+F_{1}\right)=-4[(B-C)(A+2 B+C)+A B]<0$. By (6.8) it follows that $A^{3} B$ is decreasing. Further,

$$
\frac{d}{d t} \ln \left(A^{3} B\right)=-8 \frac{F_{3}}{(A B C)^{2}}[(B-C)(A+2 B+C)+A B] \sim-8 \frac{A}{B^{2} C^{2}}(2 B-C)
$$

and

$$
\frac{d}{d t} B \sim-6 \frac{A^{2}}{B C^{2}} \text { as } t \rightarrow T
$$

As $B>0$, we must have

$$
\int^{T} \frac{A^{2}}{B C^{2}}<\infty
$$

It follows that

$$
\int^{T} \frac{A}{B^{2} C^{2}}(2 B-C)<2 \int^{T} \frac{A}{B C^{2}}<\int^{T} \frac{A^{2}}{B C^{2}}<\infty .
$$

Hence there exists $\eta \in(0, \infty)$ such that $\lim _{T} A^{3} B=\eta$. Now, as $t$ tends $T$,

$$
\frac{d}{d t} A \sim \eta \frac{A^{5}}{A^{2} B^{2} C^{2}} \sim \eta A^{15}
$$

The asymptotic for $A$ follows as well as those for $B$ and $C$. $\square$

Case 2: Inequality (6.4) is satisfied. This is equivalent to $F_{1}>0$ and implies that $F_{2}<0$. Since $B \geq C$ and $F_{1}>0$, we have $A+C<B$. Since $F_{3}>0$, we have $A$ is decreasing, $B$ is increasing and $C$ is decreasing, hence both $a$ and $c$ are decreasing. Further,

$$
\frac{F_{1}}{B^{2}}=1+\frac{C^{2}}{B^{2}}-3 \frac{A^{2}}{B^{2}}-2 \frac{C}{B}-2 \frac{A}{B} \frac{C}{B}-2 \frac{A}{B}=(1-c)^{2}-3 a^{2}-2 a c-2 a .
$$

Since both $a>0$ and $c>0$ are decreasing, $\frac{F_{1}}{B^{2}}$ is increasing, hence as long as the solution exists, we have

$$
\frac{F_{1}}{B^{2}} \geq \frac{F_{1}(0)}{B_{0}^{2}}>0
$$

We also have

$$
\left|F_{2}\right|=3 B^{2}-A^{2}-C^{2}-2 B C-2 A C+2 A B \geq 2 B^{2}-2 B C+2 A B>4 A^{2} .
$$

Hence there exists $\eta \in(0, \infty)$ such that

$$
-\frac{d}{d t} C^{2}=4 \frac{F_{1}}{B^{2}} \frac{F_{2}}{A^{2}}>\eta .
$$


This implies that the solution can only exist up to some finite time $T$ at which at least one of the following must happen: $\lim _{T} A=0, \lim _{T} C=0$ or $\lim _{T} B=\infty$.

Lemma 6.7. Assume that $B \geq C$ and (6.4) holds. Then there exists $T \in(0, \infty)$ such that the solution exists on $[0, T)$ and

$$
\lim _{t \rightarrow T} A(t)=\lim _{t \rightarrow T} C(t)=0 \text { and } \lim _{t \rightarrow T} B(t)=\infty .
$$

Further, there exists $\eta_{0} \in(0, \infty)$ such that $\lim _{T} C / A=\eta_{0}$.

Proof. As

$$
\frac{d}{d t} \ln (C / A)=8 \frac{F_{2}}{A^{2} B^{2} C^{2}}(C+A)(C-A-B)>0,
$$

$C / A$ is increasing. Hence, if $\lim C=0$, then we must have $\lim A=0$.

We first show that $\lim _{t \rightarrow T} \frac{B}{C}=\infty$, i.e., $\lim _{t \rightarrow T} c=0$. Indeed, if $\lim _{T} C=0$, since $B$ is increasing, and the desired result follows. If $\lim _{T} C=C(T)>0$, it suffices to show that $\lim B=\infty$. Assume instead that $\lim _{T} B=B(T)<\infty$. Then we must have $\lim _{T} A=0$ and, as $t \rightarrow T$,

$$
F_{2} \sim-3 B^{2}+C^{2}+2 B C \text { and } F_{3} \sim-3 C^{2}+B^{2}+2 B C .
$$

Hence there exists $\eta \in(0, \infty)$ such that

$$
\frac{d}{d t} A^{2} \sim-4 \frac{(B-C)^{2}(B+3 C)(3 B+C)}{B^{2} C^{2}} \sim \eta .
$$

It follows that $A^{2} \sim \eta(T-t)$ as $t$ tends to $T$. Also, as $t$ tends to $T$,

$$
F_{1} \sim(B-C)^{2}
$$

so

$$
\frac{d}{d t} C \sim-2 \frac{1}{\eta(T-t)} \frac{(B-C)^{3}(3 B+C)}{B^{2} C^{2}} \sim-\frac{\eta^{\prime}}{T-t},
$$

for some $\eta^{\prime} \in(0, \infty)$. This contradicts the fact that $C>0$. So $\lim _{T} B=\infty$ and $\lim _{T} B / C=\infty$.

Next we show that $\lim _{T} C=0\left(\lim _{T} A=0\right.$ follows). If not, we have $\lim _{T} C=$ $C(T)>0$ and this implies that $\lim _{T} B=\infty$. Then, since $A$ is decreasing, we have

$$
F_{1} \sim B^{2}, F_{2} \sim-3 B^{2} \text { and } F_{3} \sim B^{2} .
$$

So

$$
\frac{d}{d t} \ln B \sim \frac{2}{C(T)^{2}} \frac{B^{2}}{A^{2}} \text { and } \frac{d}{d t} C \sim-\frac{6}{C(T)} \frac{B^{2}}{A^{2}} .
$$

Since $\lim _{T} B=\infty$, we must have $\int^{T} \frac{B^{2}}{A^{2}}=\infty$. This contradicts the fact that $C>0$. So we have $\lim _{T} C=0$ as desired.

By (6.8)-(6.9), if $\alpha<\frac{1}{3}$ and $t$ is close enough to $T, A^{\alpha} B$ is increasing, but $\lim A=0$, so $\lim B=\infty$. 
Finally, we prove that $\lim _{T} \frac{C}{A} \in(0, \infty)$. We already know from $(6.11)$ that $C / A$ is increasing. On the one hand, we have

$$
\frac{d}{d t} \ln \frac{C}{A} \sim \frac{24 B}{A^{2} C^{2}}(A+C) \leq \eta \frac{B^{2}}{A^{2} C}
$$

for some $\eta \in(0, \infty)$. On the other hand,

$$
-\frac{d}{d t} C \sim 6 \frac{B^{2}}{A^{2} C}
$$

Hence

$$
\int^{T} \frac{B^{2}}{A^{2} C}<\infty
$$

It follows that $\ln \frac{C}{A}$ is bounded from above.

LEMma 6.8. Assume that $B \geq C$ and (6.4) holds. There are constants $\eta_{1}, \eta_{2}, \eta_{3} \in$ $(0, \infty)$ such that, as tends to $T$,

$$
A(t) \sim \eta_{1}(T-t)^{3 / 14}, B(t) \sim \eta_{2}(T-t)^{-1 / 14} \text { and } C(t) \sim \eta_{3}(T-t)^{3 / 14} .
$$

Proof. We first show that $\lim _{T} A^{\frac{1}{3}} B \in(0, \infty)$. By (6.8)-(6.9) with $\alpha=\frac{1}{3}$, we have

$$
\frac{d}{d t}\left(A^{\frac{1}{3}} B\right)=\frac{2 A^{\frac{1}{3}} B F_{3}}{(A B C)^{2}}\left(\frac{1}{3} F_{2}+F_{1}\right) \sim-\frac{8}{3} \frac{A^{\frac{1}{3}} B F_{3}}{A^{2} B^{2} C^{2}}(B C+2 A B)<0,
$$

for $t$ close enough to $T$, so we only need to show that $\lim _{T} A^{\frac{1}{3}} B>0$. We have

$$
\frac{d}{d t} \ln \left(A^{\frac{1}{3}} B\right) \sim-\frac{8}{3} \frac{F_{3}}{A^{2} B^{2} C^{2}}(B C+2 A B) \sim-\eta \frac{B}{A^{2} C},
$$

for some constant $\eta \in(0, \infty)$ (here, we use that $\lim _{T} C / A \in(0, \infty)$ ). Further,

$$
\frac{d}{d t} A=\frac{2 A F_{2} F_{3}}{(A B C)^{2}} \sim-6 \frac{B^{2}}{A C^{2}} .
$$

Since $A$ is bounded, we must have $\int^{T} \frac{B^{2}}{A C^{2}}<\infty$. Hence $\int^{T} \frac{B}{A^{2} C}<\infty$ as well. This implies $\lim _{T} A^{\frac{1}{3}} B=\eta>0$ as desired. It follows that $A B^{3}$ and $C B^{3}$ have positive finite limits as $t$ tends to $T$ and we can proceed as in case 1 to obtain the asymptotic of $A, B$ and $C$ when $t$ tends to $T$. $\square$

Case 3: Neither (6.2) nor (6.4) are ever satisfied along the flow Assume the solution exists on the interval $[0, T)(T$ can be $\infty$ here). The third case is the case when

$$
\forall t \in[0, T), \quad \frac{1}{3}\left(2 \sqrt{1-c+c^{2}}-1-c\right) \leq a \leq 1-c+2 \sqrt{1-c} .
$$

Recall that (6.2) is equivalent to $F_{2}>0$ and (6.4) is equivalent to $F_{1}>0$. Hence we have $F_{1}, F_{2} \leq 0$. Since $F_{1}+F_{2}<0$, at least one of them is strictly negative in this case. We first notice that $F_{3} \geq A^{2}>0$. It follows that $A$ and $B$ are non-increasing, and $C$ is non-decreasing. So we have

$$
C_{0} \leq C \leq B \leq B_{0}
$$

and $B, C$ have finite positive limits when $t$ tends to $T$.

Lemma 6.9. Assume $B \geq C$. 
- If $a_{0}=A_{0} / B_{0}, c_{0}=C_{0} / B_{0}$ satisfy

$$
a \geq 1-c .
$$

then this inequality is satisfied for all $t \in[0, T)$ and there exists a time $t_{1} \in$ $[0, T)$ such that $(6.2)$ holds for all $t \in\left(t_{1}, T\right)$.

- If (6.12) holds then, for all $t \in[0, T), A+C<B$, that is, $a<1-c$.

Proof. Observe that for any $c \in[0,1]$,

$$
1-c \geq \frac{(1-c)^{2}}{2 \sqrt{1-c+c^{2}}+1+c}=\frac{1}{3}\left(2 \sqrt{1-c+c^{2}}-1-c\right) .
$$

By Lemma 6.3, this shows that $a \geq 1-c$ implies $F_{1} \leq 0$. Assume that $A+C \geq B$ at some time $t$. Then, at that time, (6.5) and (6.6) show that $\frac{d}{d t} \ln (A / B) \geq 0$ and $\frac{d}{d t} \ln (C / B) \geq 0$. This proves that the inequality $a \geq 1-c$ is preserved by the flow.

Assume now that (6.12) holds. Then both $B$ and $C$ are monotone and have positive finite limits as $t$ tends to $T$. If there exists a time $t_{0}$ such that $a \geq 1-c$ at time $t_{0}$, then $a=A / B$ is non-decreasing for $t \geq t_{0}$. This means that the solution must exist for all time, i.e., $T=\infty$. However,

$$
\left|F_{1}\right|=3 A^{2}-(B-C)^{2}+2 A C+2 A B \geq 2 A(A+B+C),
$$

so $\frac{d}{d t} B<-\eta$ with $\eta$ a positive constant. This contradicts $T=\infty$ and thus shows that $A+C<B$, for all $t \in[0, T)$.

Finally, since $a \geq 1-c$ is preserved, implies $F_{1} \geq 0$, and is incompatible with (6.13), it follows that if $a_{0} \geq 1-c_{0}$ then there exists $t_{1}$ such that $F_{2}>0$, that is (6.2) holds (it then holds for all $t>t_{1}$ by Lemma 6.2).

Lemma 6.10. Assume $B \geq C$ and (6.12). Then we have

$$
\lim _{t \rightarrow T} A(t)=0 \text { and } \lim _{t \rightarrow T} B(t)=\lim _{t \rightarrow T} C(t)=k \in(0, \infty) .
$$

Proof. Observe that $F_{2}+F_{3}=2(A+B-C)(A-B+C)<0$. It follows that $\left|F_{2}\right|>F_{3} \geq A^{2}$. If $\lim _{T} A=A(T)>0$ then we must have $T=\infty$. However, $\frac{d}{d t} A<-\eta$ with $\eta$ a positive constant so the solution can only exist up to some finite time, a contradiction. This shows that $\lim _{T} A=0$. Since $0 \leq B-C \leq A$, we also have $\lim _{T}(B-C)=0$. $\mathrm{Q}$

Lemma 6.11. Assume $B \geq C$ and (6.12). Then $T<\infty$, and

$$
\lim _{t \rightarrow T} \frac{2 k A}{(B-C)^{2}}=1 \text {. }
$$

Proof. First we observe that the inequality $4 A B \geq(B-C)^{2}$ follows easily from the fact that

$$
a \geq \frac{1}{3}\left(2 \sqrt{1-c+c^{2}}-1-c\right)=\frac{(1-c)^{2}}{2 \sqrt{1-c+c^{2}}+1+c} .
$$

For any positive number $\alpha$, we have

$$
\frac{d}{d t} \ln A=\frac{2}{A^{2} B^{2} C^{2}} F_{2} F_{3} \text { and } \frac{d}{d t} \ln (B-C)=\frac{2}{A^{2} B^{2} C^{2}} F_{1} Y .
$$


It follows that

$$
\frac{d}{d t} \ln \frac{A}{(B-C)^{\alpha}}=\frac{2}{A^{2} B^{2} C^{2}}\left(F_{2} F_{3}-\alpha F_{1} Y\right),
$$

where $Y=A^{2}+B^{2}+C^{2}+6 B C+2 A B+2 A C$. Since $B-C>A$ and $\lim _{T} A=0$, as $t$ tends to $T$, we have

$$
\begin{gathered}
F_{2}=-(2 A+C+3 B)(B-C)+A^{2}=-4 k(B-C)+O\left((B-C)^{2}\right), \\
F_{3}=(B-C)(B+3 C+2 A)+A^{2}=4 k(B-C)+O\left((B-C)^{2}\right), \\
-F_{1}=2 A B+2 A C-(B-C)^{2}+3 A^{2}=4 k A-(B-C)^{2}+O(A(B-C)),
\end{gathered}
$$

and

$$
Y=8 k^{2}+O((B-C)) .
$$

In this estimates, we have used the fact that $0 \leq k-C, B-k \leq B-C$. This yields

$$
F_{2} F_{3}-\alpha F_{1} Y=8 \alpha k^{2}\left[4 k A-(1+(2 / \alpha))(B-C)^{2}\right]+O\left[A(B-C)+(B-C)^{3}\right] .
$$

We first show that for any $\epsilon>0$ there exists $t_{0}$ such that

$$
\forall t \in\left[t_{0}, T\right), \frac{4 k A}{(B-C)^{2}} \geq 2-\epsilon .
$$

Otherwise, there exists $\epsilon>0$ such that for any $t_{0}$ there exists $t_{1} \in\left(t_{0}, T\right)$ such that $4 k A /\left.(B-C)^{2}\right|_{t=t_{1}}<2-\epsilon$. Taking $t_{0}$ large enough, $\alpha=2$ in (6.14) and using (6.15), it follows that we must have $4 k A /(B-C)^{2}<2-\epsilon$ for all $t \in\left[t_{1}, T\right)$. Now, taking $\alpha=2+\delta(\epsilon)$ in (6.14), we obtain that $A /(B-C)^{2+\delta(\epsilon)}$ is decreasing if $2-\epsilon<1+\frac{2}{2+\delta(\epsilon)}$. This contradicts the fact that $A /(B-C)^{2} \geq 1 / 4 B$.

Now, as $t$ tends to $T$,

$$
\left|F_{1}\right|=2 A C+2 A B-(B-C)^{2}+3 A^{2} \geq k A .
$$

Hence, there exists $\eta \in(0, \infty)$ such that

$$
\frac{d}{d t} C=\frac{2}{B^{2} C^{2}} \frac{\left|F_{1}\right|}{A} \times \frac{\left|F_{2}\right|}{A}>\eta
$$

As $C$ is bounded from above by $B_{0}$, this implies $T<\infty$.

Next we show that there exists $t_{0} \in([0, T)$ such that

$$
\forall t \in\left[t_{0}, T\right), \frac{k A}{(B-C)^{2}}<1 .
$$

Otherwise, for any $t_{0}$ there exists $t_{1} \in\left(t_{0}, T\right)$ such that $k A /\left.(B-C)^{2}\right|_{t=t_{1}} \geq 1$. Using (6.14)-(6.15), it is easy to see the $k A /(B-C)^{2} \geq 1$ for all $t \in\left[t_{1}, T\right)$. Now take $\alpha=1$ in (6.14)-(6.15) to see that $A /(B-C)$ is increasing on $\left(t_{2}, T\right)$ for some $t_{2} \in\left(t_{1}, T\right)$. It follows that there exists $\eta \in(0, \infty)$ such that

$$
-\frac{d}{d t} \ln A \sim \eta \frac{(B-C)^{2}}{A^{2}}<\eta .
$$


As $T<\infty$, this contradicts $\lim _{T} A=0$.

We now show that for any $\epsilon>0$ there exists $t_{0}$ such that

$$
\forall t \in\left[t_{0}, T\right), \frac{4 k A}{(B-C)^{2}} \leq 2+\epsilon .
$$

Otherwise, there exists $\epsilon>0$ such that for any $t_{0}$ there exists $t_{1} \in\left(t_{0}, T\right)$ such that $4 k A /\left.(B-C)^{2}\right|_{t=t_{1}}>2+\epsilon$. Taking $t_{0}$ large enough, $\alpha=2$ in (6.14) and using (6.15), it follows that we must have $4 k A /(B-C)^{2}>2+\epsilon$ for all $t \in\left[t_{1}, T\right)$. Now using $\alpha=2-\delta(\epsilon)$ in (6.14)-(6.15), we find that $A /(B-C)^{2-\delta(\epsilon)}$ is non-decreasing if $2+\epsilon>1+\frac{2}{2-\delta(\epsilon)}$. This contradicts $A /(B-C)^{2} \leq 3 / 4 k$. $\square$

Using $2 k A \sim(B-C)^{2}$ in the original ODEs (6.1), we easily find the asymptotic behaviors of $A, B$ and $C$. Namely, as $t \rightarrow T$, we have

$$
A \sim \frac{64}{k}(T-t), \quad B \sim k+4 \sqrt{2} \sqrt{T-t} \text { and } C \sim k-4 \sqrt{2} \sqrt{T-t} .
$$

Putting together the different cases yields the following statement.

THEOREM 6.12. Let $g(t)$ be the solution of $(+\mathrm{XCF})$ on $\mathrm{SL}(2, \mathbb{R})$ with given initial data $A_{0}, B_{0}, C_{0}>0, B_{0} \geq C_{0}$, in a Milnor frame $f_{1}, f_{2}, f_{3}$. Then there exists a finite positive time $T_{b}$ such that $g(t)$ exists for all $t \in\left[0, T_{b}\right)$. Moreover, we have:

1. If there is a time $t$ such that $A \geq B-C$ then there are constant $\eta_{i} \in(0, \infty)$ such that, as $t \rightarrow T_{b}$,

$$
A \sim \eta_{1}\left(T_{b}-t\right)^{-\frac{1}{14}}, B \sim \eta_{2}\left(T_{b}-t\right)^{\frac{3}{14}}, \text { and } C \sim \eta_{3}\left(T_{b}-t\right)^{\frac{3}{14}} .
$$

2. If there is a time $t$ such that $A<\frac{1}{3}\left(2 \sqrt{B^{2}-B C+C^{2}}-B-C\right)$ then there are constant $\eta_{i} \in(0, \infty)$ such that, as $t \rightarrow T_{b}$,

$$
A \sim \eta_{1}\left(T_{b}-t\right)^{\frac{3}{14}}, B \sim \eta_{2}\left(T_{b}-t\right)^{-\frac{1}{14}}, \text { and } C \sim \eta_{3}\left(T_{b}-t\right)^{\frac{3}{14}} .
$$

3. If for all $t, \frac{1}{3}\left(2 \sqrt{B^{2}-B C+C^{2}}-B-C\right) \leq A<B-C$, then as $t \rightarrow T_{b}$,

$$
\begin{aligned}
& A \sim \frac{64}{C\left(T_{b}\right)}\left(T_{b}-t\right), B \sim C\left(T_{b}\right)+4 \sqrt{2\left(T_{b}-t\right)}, \text { and } C \sim C\left(T_{b}\right)-4 \sqrt{2\left(T_{b}-t\right)} \\
& \text { with } C\left(T_{b}\right) \in(0, \infty) .
\end{aligned}
$$

REMARK 6.3. A priori, the statement above does not imply that the case when

$$
\frac{1}{3}\left(2 \sqrt{B^{2}-B C+C^{2}}-B-C\right) \leq A<B-C
$$

holds for all $t \in[0, T)$ really occurs since it is possible that no solutions satisfy $\frac{1}{3}\left(2 \sqrt{B^{2}-B C+C^{2}}-B-C\right) \leq A<B-C$ for all $t \in\left[0, T_{b}\right)$. In [CGSC09], we will show by different techniques that this case really occurs but only for initial condition on an hypersurface. See the discussion below.

REMARK 6.4. In case (3), we have

$$
\lim _{t \rightarrow T_{b}} K\left(f_{2} \wedge f_{3}\right)=-\frac{2}{C\left(T_{b}\right)}
$$


and the two other sectional curvatures vanish. If $M$ is compact, then there is collapse in the $f_{1}$ direction.

It is quite clear that Theorem 6.12 implies Theorem 6.1 and give a more precise and technical description of the asymptotic behavior of $(+\mathrm{XCF})$ on $\mathrm{SL}(2, \mathbb{R})$. More precisely, the initial condition space

$$
Q=\left\{(a, b, c) \in \mathbb{R}^{3}: a>0, b \geq c>0\right\}
$$

can be partitioned into $Q=Q_{1} \cup Q_{2} \cup S_{0}$ with

$$
\begin{gathered}
\{(a, b, c) \in Q: a \geq b-c\} \subset Q_{1}, \\
\left\{(a, b, c) \in Q: 3 a<2 \sqrt{b^{2}-b c+c^{2}}-(b+c)\right\} \subset Q_{2},
\end{gathered}
$$

and

$$
S_{0} \subset\left\{(a, b, c) \in Q:\left(2 \sqrt{b^{2}-b c+c^{2}}-(b+c)\right) \leq 3 a<3(b-c)\right\} .
$$

By Theorem 6.12(1)-(2), for initial condition in $Q_{1}$ (resp. $Q_{2}$ ) the distance function associated to the metric $\bar{g}=\left(C_{0} / C(t)\right) g(t)$ clearly converges uniformly on compact sets to the distance function associated with a sub-Riemannian structure of the form $\gamma_{2} f_{2} \otimes f_{2}+\gamma_{3} f_{3} \otimes f_{3}$ (resp. $\left.\gamma_{1} f_{1} \otimes f_{1}+\gamma_{3} f_{3} \otimes f_{3}\right)$. In fact, as stated in Theorem 6.12 , it suffices that $g(t)$ enters the region $Q_{1}$ or $Q_{2}$ at some time $t \geq 0$. Hence, we can define $Q_{1}$ (resp. $Q_{2}$ ) to be the set of initial conditions in $Q$ such that $g(t)$ enters $\{a \geq b-c\}$ (resp. $\left\{3 a<2 \sqrt{b^{2}-b c+c^{2}}-(b+c)\right\}$ ) and $S_{0}=Q \backslash\left(Q_{1} \cup Q_{2}\right)$. From this discussion it seems very plausible that $S_{0}$ is simply a hypersurface separating $Q_{1}$ and $Q_{2}$. However, we have not been able to prove this by arguments similar to those used above. In [CGSC09], we prove that $S_{0}$ is indeed a surface separating the open sets $Q_{1}, Q_{2}$ by reducing the ODEs to a two dimensional one and using the fact that the orbit structure of 2-dimensional ODEs can be understood much better than in higher dimension.

\section{REFERENCES}

[BBI01] D. Burago, Y. Burago, and S. Ivanov, A course in metric geometry, volume 33 of "Graduate Studies in Mathematics", American Mathematical Society, Providence, RI, 2001.

[Buc06] J. A. BuCKLAND, Short-time existence of solutions to the cross curvature flow on 3-manifolds, Proc. Amer. Math. Soc., 134:6 (2006), pp. 1803-1807 (electronic).

[CGSC09] X. Cao, J. Guckenheimer, and L. Saloff-Coste, The backward behavior of the Ricci and cross curvature flow on $S L(2, R)$, Comm. Anal. Geom., 17:4 (2009), pp. $777-796$.

[CH04] B. CHOW AND R. S. HAMilton, The cross curvature flow of 3-manifolds with negative sectional curvature, Turkish J. Math., 28:1 (2004), pp. 1-10.

[CK04] B. ChOw AND D. KNOPF, The Ricci flow: an introduction, volume 110 of Mathematical Surveys and Monographs, American Mathematical Society, Providence, RI, 2004.

[CNSC08] X. CAO, Y. Ni, And L. SAloff-Coste, Cross curvature flow on locally homogeneous three-manifolds. I, Pacific J. Math., 236:2 (2008), pp. 263-281.

[CSC09] X. CAO And L. Saloff-Coste, Backward Ricci flow on locally homogeneous 3manifolds, Comm. Anal. Geom., 17:2 (2009), pp. 305-325.

[Gli08] D. GLickenstein, Riemannian groupoids and solitons for three-dimensional homogeneous Ricci and cross-curvature flows, Int. Math. Res. Not. IMRN, (12):Art. ID rnn034, 49, 2008. 
[Ham82] R. S. HAmilton, Three-manifolds with positive Ricci curvature, J. Differential Geom., 17:2 (1982), pp. 255-306.

[IJ92] J. ISENBERG AND M. JACKSON, Ricci flow of locally homogeneous geometries on closed manifolds, J. Differential Geom., 35:3 (1992), pp. 723-741.

[IJL06] J. IsEnBerg, M. JACKSON, AND P. Lu, Ricci flow on locally homogeneous closed 4manifolds, Comm. Anal. Geom., 14:2 (2006), pp. 345-386.

[JSC87] D. JERISON AND A. SÁNCHEZ-CALLE, Subelliptic, second order differential operators, in "Complex analysis, III (College Park, Md., 1985-86)", volume 1277 of "Lecture Notes in Math.", pp. 46-77. Springer, Berlin, 1987.

[Mil76] J. Milnor, Curvatures of left invariant metrics on Lie groups, Advances in Math., 21:3 (1976), pp. 293-329.

[Mon02] R Montgomery, A tour of subriemannian geometries, their geodesics and applications, volume 91 of "Mathematical Surveys and Monographs", American Mathematical Society, Providence, RI, 2002. 